\title{
Dissection-independent production of Plasmodium sporozoites from whole mosquitoes
}

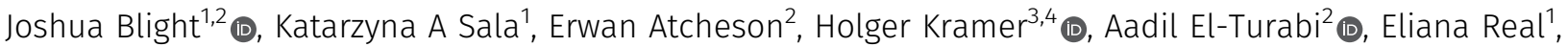 \\ Farah A Dahalan¹, Paulo Bettencourt ${ }^{2}$ (D) Emma Dickinson-Craig ${ }^{2}$, Eduardo Alves ${ }^{2}$, Ahmed M Salman ${ }^{2}$, Chris J Janse $^{5}$ (D, \\ Frances M Ashcroft ${ }^{3}$ (D), Adrian VS Hill ${ }^{2}$ (D), Arturo Reyes-Sandoval ${ }^{2,6}$ (D), Andrew M Blagborough ${ }^{1,7}$, Jake Baum $^{1}$ (D)
}

Progress towards a protective vaccine against malaria remains slow. To date, only limited protection has been routinely achieved following immunisation with either whole-parasite (sporozoite) or subunit-based vaccines. One major roadblock to vaccine progress, and to pre-erythrocytic parasite biology in general, is the continued reliance on manual salivary gland dissection for sporozoite isolation from infected mosquitoes. Here, we report development of a multi-step method, based on batch processing of homogenised whole mosquitoes, slurry, and density-gradient filtration, which combined with free-flow electrophoresis rapidly produces a pure, infective sporozoite inoculum. Human-infective Plasmodium falciparum and rodent-infective Plasmodium berghei sporozoites produced in this way are two- to threefold more infective than salivary gland dissection sporozoites in in vitro hepatocyte infection assays. In an in vivo rodent malaria model, the same $P$. berghei sporozoites confer sterile protection from mosquito-bite challenge when immunisation is delivered intravenously or $60-70 \%$ protection when delivered intramuscularly. By improving purity, infectivity, and immunogenicity, this method represents a key advancement in capacity to produce researchgrade sporozoites, which should impact delivery of a wholeparasite based malaria vaccine at scale in the future.

DOI 10.26508/Isa.202101094 | Received 14 April 2021 | Revised 24 May 2021 | Accepted 25 May 2021 | Published online 16 June 2021

\section{Introduction}

A vaccine against malaria is still urgently required to address the nearly half a million deaths caused by the disease each year (1). Developmental malaria vaccines currently focus on two distinct strategies, either recombinant production and immunisation of dominant surface antigens from the Plasmodium parasite, or labour-intensive production, purification, and immunisation with live parasites that are attenuated or delivered under drug coverage (2). The most advanced of these vaccines have focussed on preventing the pre-erythrocytic stages of parasite infection, targeting the infectious sporozoite form between its injection into the skin by the feeding mosquito and its first destination within the human liver (2). Pre-erythrocytic subunit vaccines to date have largely been based on the dominant sporozoite surface antigen, circumsporozoite protein (CSP) (3). CSP has been the subject of intensive investigation for more than $40 \mathrm{yr}$. Its most recent formulation within the RTS,S vaccine confers moderate protection following challenge in phase 3 trials, although long-term efficacy remains uncertain (4). Sporozoite-based vaccination by controlled mosquito-bite with drug coverage on the other hand, has consistently been shown to confer sterile (complete) protection (5). Mosquito-based delivery for large-scale vaccination is, however, impractical. The best alternative to this is live-attenuated sporozoite vaccination delivered directly $(6,7,8,9,10)$. By producing parasites that arrest prematurely in the liver $(11,12)$, both humoral and cellular immunity can develop, offering long-term protection $(8,13)$. Indeed, the most advanced of these, called PFSPZ ( $P$. falciparum sporozoite) (14), can confer sterile protection under controlled clinical conditions. A limitation to its utility remains the requirement for i.v. delivery and use of substantial sporozoite numbers, as high as 270,000 sporozoites per immunisation, and the requirement for multiple rounds of immunisation (typically one prime and three boosts) (2, 8, 13). PfSPZ has shown moderate efficacy (up to $52 \%$ at $24 \mathrm{wk}$ ) against naturally transmitted malaria (15), although vaccine efficacy long-term is still pending. Thus, while showing great promise PfSPZ, such as RTS,S, still falls short of the preferred efficacy aspired to for future malaria vaccines (16).

Live-attenuated whole sporozoite vaccination approaches rely on generating large amounts of pure and aseptic parasites for clinical grade vaccine manufacture (13). This is a considerable bottleneck to vaccine design, testing, and implementation in terms of scale, time, and cost. At present, sporozoites can only be isolated

\footnotetext{
${ }^{1}$ Department of Life Sciences, Imperial College London, Sir Alexander Fleming Building, London, UK ${ }^{2}$ The Jenner Institute, University of Oxford, Old Road Campus Research Building, Oxford, UK ${ }^{3}$ Department of Physiology, Anatomy and Genetics, Henry Wellcome Building for Gene Function, University of Oxford, Oxford, UK ${ }^{4}$ Medical Research Council London Institute of Medical Sciences, Imperial College London, Hammersmith Hospital, London, UK ${ }^{5}$ Department of Parasitology, Leiden Malaria Research Group, Center of Infectious Diseases, Leiden University Medical Center, (LUMC, L4-Q), Leiden, The Netherlands ${ }^{6}$ Instituto Politécnico Nacional, Mexico City, Mexico ${ }^{7}$ Department of Pathology, University of Cambridge, Cambridge, UK
}

Correspondence: jake.baum@imperial.ac.uk 
from infected mosquitoes via salivary gland dissection (SGD). As well as the obvious challenges this presents to vaccine development, difficulties with sporozoite isolation have also held back general understanding of sporozoite biology. While substantial advances have been made in development of experimental in vitro hepatocyte models of infection $(17,18)$, these are much less accessible and less reproducible when compared to routine culture and study of blood-stage parasites. SGD requires in vivo parasite development in the mosquito followed by manual dissection of the salivary glands 15-21 d post infected blood feed. Originally described in 1964 (19) with only minor variations since (17, 20, 21, 22), the dissection method involves mosquito decapitation, gland removal and homogenisation to release sporozoites. Dissection in this way is time-consuming, taking a skilled technician an hour or so to dissect 100 or more glands to a reasonable standard. With total extraction time being a critical factor for subsequent sporozoite viability (23), there is a relatively low upper limit for attaining live, infectious sporozoites. Furthermore, SGD sporozoites retain a considerable amount of mosquito-originating debris (24). Some of this debris, for example, salivary gland-associated proteins, have been shown to inhibit sporozoite motility, which is critical for liver cell infection (25). Other mosquito-derived contaminating proteins have been shown to modulate immune response in vivo (26), potentially affecting vaccinations. Likewise, the time taken, and contamination carried over, places limits on the infectivity and development of Plasmodium sporozoites in vitro $(20,24,27)$. Rates of cell infection with in vitro hepatocyte (primary or hepatoma) cultures using SGD are typically $<1 \%$ using the rodent malaria model Plasmodium berghei $(28,29,30,31)$ and $<2 \%$ for human Plasmodium falciparum sporozoites $(18,20,22,24,32,33)$. These limitations have been a major impediment to in vitro studies and for screens that rely on high rates of infection.

Several previous attempts have sought to improve throughput and purity of whole sporozoite preparation. Methods aimed at bypassing SGD have included centrifugation through glass wool (34) and compression between glass plates (35). These alternative methods have not substantially improved parasite purity, even when combined with density gradients $(33,36,37,38,39,40)$. While the addition of gradients increase sporozoite yield, the final output is still contaminated with mosquito debris $(25,41)$. Other methods trialled for sporozoite isolation have included ion exchange chromatography $(41,42)$, and later free-flow electrophoresis (FFE) (43). FFE is a liquid form of electrophoresis commonly used to separate organelles under native conditions based on net surface charge (44). The poor yields or complexity of these two methods has limited interest in their scaled usage. This is despite significant, although unexplored, recent developments in FFE technology in particular (http://www.ffeservice.com). To date, the only scaled means for manufacture of a clinical grade vaccine has therefore relied on enlisting multiple skilled manual dissectors combined with rearing of parasites within aseptic mosquitoes. Automated aids for dissection have been described, although these still require some manual mosquito manipulation (45 Preprint).

Obtaining malaria sporozoites therefore remains a major challenge for improving understanding of basic parasite transmission biology, and a significant hurdle for scalable and reproducible production of whole sporozoites for direct vaccination. In response to this challenge, here we describe a multi-step method that successfully purifies both rodent $P$. berghei and human $P$. falciparum sporozoites from whole mosquitoes in a batch process, based on an optimized combination of homogenisation, size exclusion, density and charge. This stepwise approach facilitates processing of hundreds of mosquitoes per hour, rapidly harvesting pure sporozoites, and can be adapted to produce effectively contaminant-free, vaccine-grade sporozoites by a single trained technician. The sporozoites isolated by this process show excellent infectivity both in vitro and in vivo and offer sterile protection in a rodent model when given as a live-attenuated vaccine or $60-70 \%$ protection when administered via intra-muscular delivery. Being dissection-independent, this process can facilitate the rapid and scalable manufacture of Plasmodium sporozoites to advance preerythrocytic Plasmodium research and as an enabling technology that can be harnessed for delivery of future whole-parasite based malaria vaccines.

\section{Results}

\section{Rapid, dissection-independent, isolation of sporozoites from whole mosquitoes}

The challenges of obtaining sporozoites for malaria research by SGD are a major impediment to improving understanding of the liver stages and development of effective whole-parasite vaccination $(2,24,30,31,32,46,47,48,49)$. Sporozoite isolation by SGD is a low-throughput and labour-intensive procedure which produces sporozoites of mixed purity, often contaminated with mosquitoassociated material. To address this need, we have developed a stepwise method for the purification of sporozoites from whole mosquitoes without requiring manual dissection (Fig 1A).

Our method consists of three discrete steps, with capacity to process up to 1,000 mosquitoes at a time (over a 2-h window) by a single individual. Whole mosquitoes were homogenised to release sporozoites and filtered sequentially through 100-10- $\mu \mathrm{m}$ filters. The filtered mosquito homogenate/Mash (M) was then pre-purified by density centrifugation using Accudenz (MA), as previously described (24), to remove larger mosquito-associated debris from sporozoites. The sporozoite layer was subsequently purified by FFE, based on total net charge (MAF) (Fig 1B and Table S1) using a continuous zone electrophoresis (CZE) mode (see the Materials and Methods section). Output consisted of 96 fractions with a peak sporozoite fraction, as represented by purification of rodent malaria $P$. berghei mCherry sporozoites assessed by light microscopy (Fig 1C) or fluorescent plate reader for mCherry fluorescence (Fig S1A).

$P$. berghei sporozoites produced according to this multi-step method demonstrated reproducible separation, independent of initial sporozoite quantity. Most sporozoites separated into a single fraction, with a characteristic tail in the distribution that lengthened as sporozoite dose load increased (Fig S1B and C). This peak fraction was used for all subsequent experiments. A single run resulted in a final 51-65\% loss in yields compared with the starting material; however, the FFE step was responsible for only a $30 \%$ loss of sporozoites from the preceding step in the protocol. The 
A.

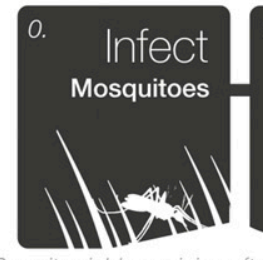

Parasite yield remaining after eac

B.

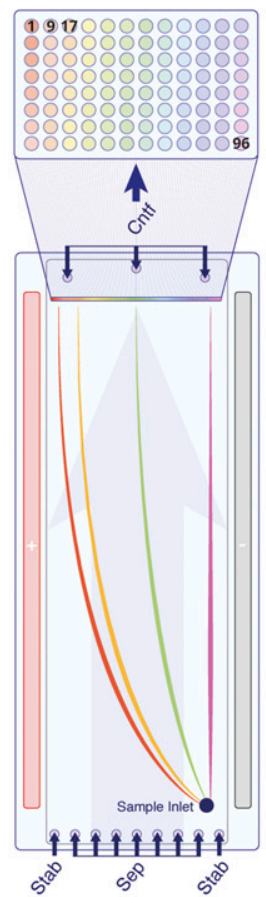

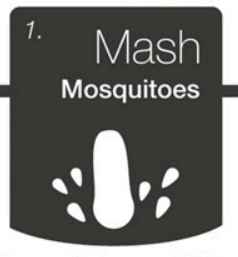

M

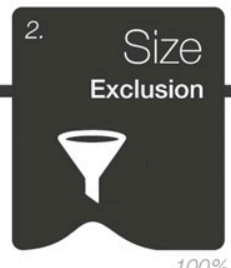

C.

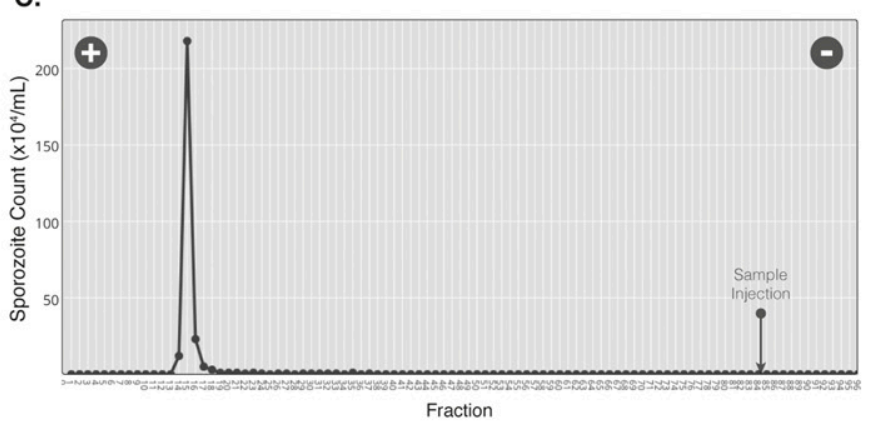

F.
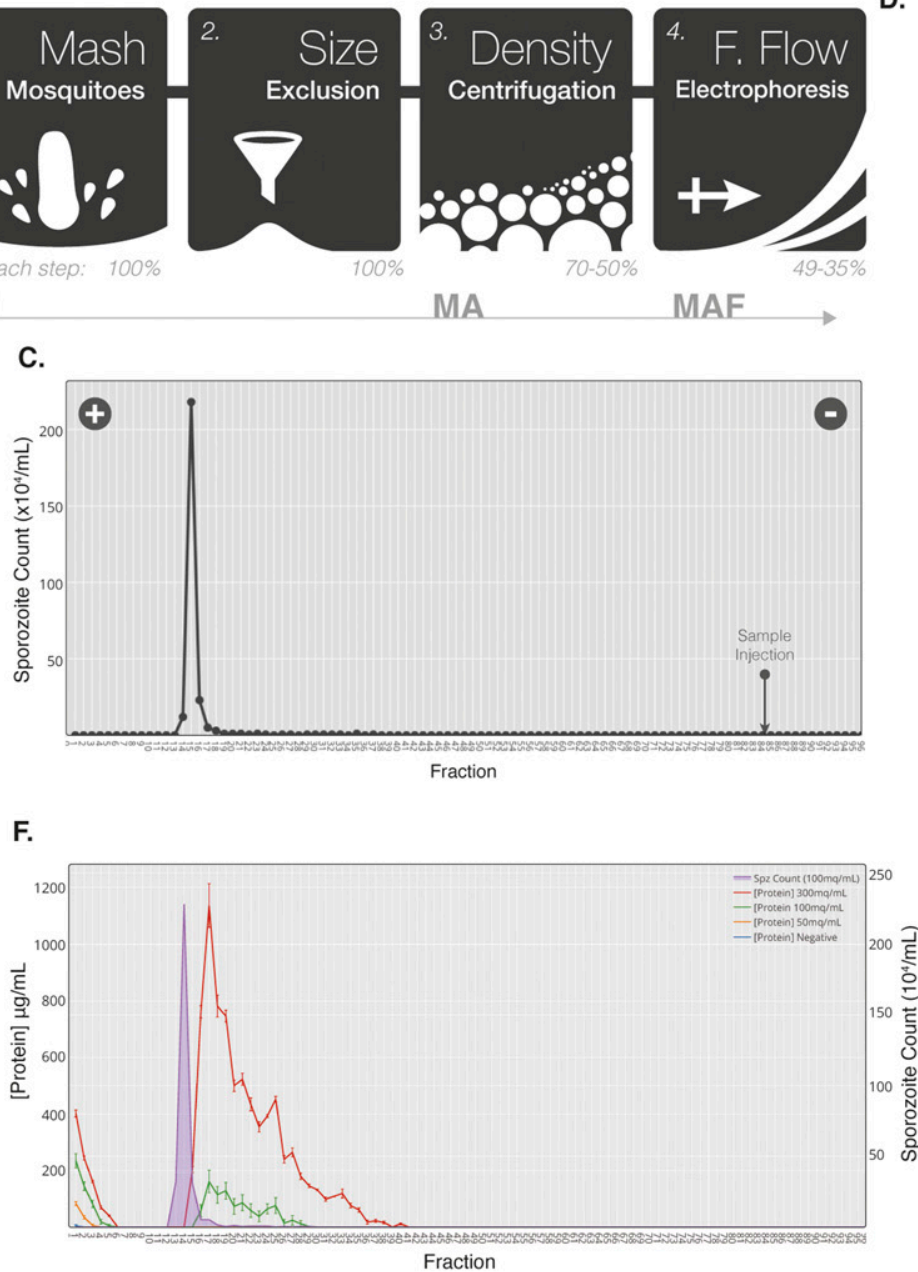

MA

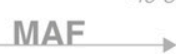

MAF

D.

E

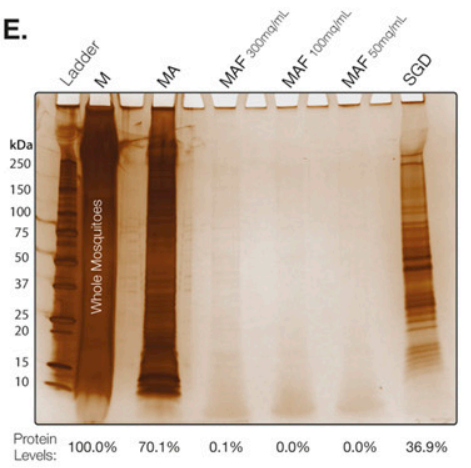

G.

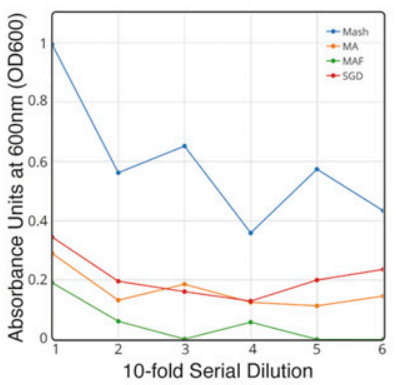

H.

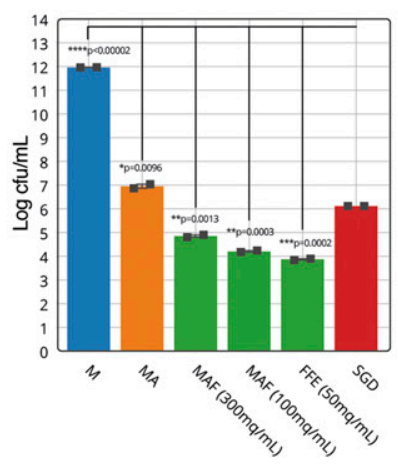

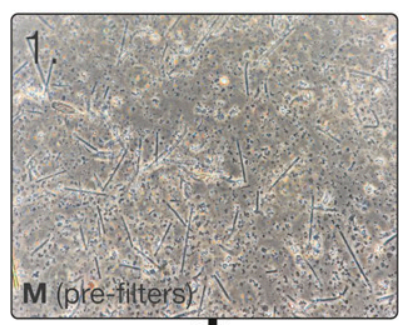

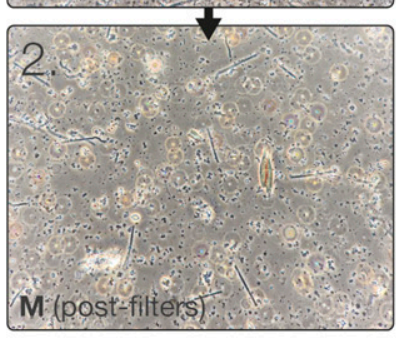

3.

\section{MA}

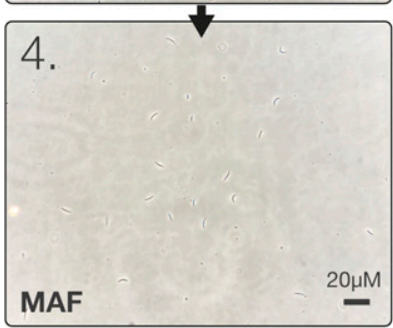

I.

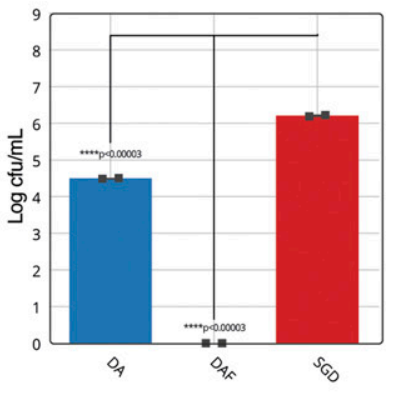

Figure 1. Development of a stepwise process for purification of sporozoites from whole mosquitoes.

(A) Schematic of key steps in the sporozoite purification process. (B) Schematic representation of sample separation by continuous zone electrophoresis mode. An electrophoretic buffer is run through a chamber $0.5 \mathrm{~mm}$ thick with a voltage applied across the flow. Sample added to the start of the chamber is carried vertically up the length of the chamber (pale blue arrow) as a voltage is applied across, separating across the horizontal length of the chamber. The outflow from the chamber is separated into 96 outlets along the horizontal length of the chamber, which drop into a 96 well plate. (C) Manual sporozoite count by haemocytometer of free-flow electrophoresis (FFE) fractions from a representative MAF sporozoite separation. Point of sample injection indicated by arrow and direction of current indicated by positive and negative symbols. (D) Bright-field images of each stage of purification from whole mosquito homogenate. All stages diluted to $7 \times 10^{5} \mathrm{sporozoites} / \mathrm{ml}$. (E) Silver stain of reducing SDS-PAGE gel with uninfected mosquitoes (four MEQs) from each step of purification. Uninfected MAF lanes are from the same fraction as the sporozoite peak fraction identified by running infected mosquitoes at the same time. (F) Protein concentration in each fraction after loading uninfected mosquito MA onto the FFE machine at three doses of mosquitoes (MAF). Sporozoite distribution (purple) from infected mosquitos loaded at $100 \mathrm{mq} / \mathrm{ml}$ is marked to allow comparison of purification. (G) End point 16-h serial dilution for each step of MAF purification. Absorbance of samples in TBS was measured at $600 \mathrm{~nm}$ (OD600) $16 \mathrm{~h}$ post-inoculation ay $37^{\circ} \mathrm{C}$. All growth 
Accudenz step was responsible for up to a $50 \%$ loss in yield from its preceding step. Of note, Accudenz on its own is known to be associated with a loss of sporozoite yield of up to $50 \%$. Using this method, 500-1,000 mosquitoes could be processed by one individual in $2 \mathrm{~h}$. This same skilled user could process a few hundred mosquitoes in a similar timeframe using traditional SGD (range of 100-200 by our experienced dissectors). This represents a $5-10$-fold increase in throughput comparing MAF to SGD (incorporating Accudenz purification) with the potential for running parallel units to scale production accordingly, although without requiring additional staffing.

Given the established potential of whole sporozoites as an effective vaccine (9), we next sought to establish the purity of MAF sporozoites compared with SGD sporozoites. Initial assessment of bright-field images showed that our method successfully removed all visible mosquito-associated debris (Fig 1D). To quantitatively assess contaminants, samples were normalised by mosquito equivalents (MEQ); based on the number of mosquitoes (mq) homogenised and volume (units: $\mathrm{mq} / \mathrm{ml}$ ) as opposed to sporozoite dose, recognizing that this can vary substantially between batches. To assess the sequential reduction in protein contaminants during each step of the purification, uninfected mosquitoes were purified to determine contributing mosquito protein contaminants. MAF samples were run at three different MEQs $(300,100$, and $50 \mathrm{mq} / \mathrm{ml})$ on FFE to determine an optimal purification condition. Uninfected mosquitoes processed by MAF showed a complete absence in detectable mosquito-derived protein by silver stain when loaded onto FFE at $100 \mathrm{mq} / \mathrm{ml}$ or less. An equivalent preparation of the same number of MEQs using uninfected mosquito-derived SGD showed only a $63.1 \%$ reduction in contaminants when compared with crude input (Fig 1E). The differences in the FFE separation profile of mosquito-associated protein at the three MEQs demonstrated that at $100 \mathrm{mq} / \mathrm{ml}$ or less, all detectable protein could be effectively removed from the peak sporozoite positive FFE fractions (Fig 1F).

Analysis of the FFE output demonstrated our ability to remove abundant mosquito proteins, such as actin, as well as enriching for sporozoite proteins in the sporozoite fraction (Fig 1D-F). In addition, an identical protein purification profile was obtained when using dissected salivary glands as the starting homogenate rather than whole mosquitoes (referred to as Dissected-Accudenz-FFE; DAF) (Fig S1D), demonstrating the flexibility of our stepwise process for isolating different sub-populations of sporozoite within the infected mosquito, for example, where separation of immature haemocoel sporozoites from those in the head/salivary gland is desired.

Given that bacterial contamination is a major problem for in vitro work, we next assessed the ability of our stepwise process to separate mosquito-associated bacteria. Serial dilutions of samples normalised to equal MEQ from each stage of purification were grown for $16 \mathrm{~h}$ at $37^{\circ} \mathrm{C}$ in a non-selective tryptic soya broth medium (50) (Fig 1G). A marked reduction in bacterial growth, assessed by measuring OD600, was observed with MAF purified sporozoites. This was further confirmed by measuring bacteria colony-forming units per $\mathrm{ml}(\mathrm{cfu} / \mathrm{ml}$ ) on blood-agar plates, which showed a significant 8.1 log reduction in total bacterial load compared with a 5.9 log reduction by SGD (Figs 1H, S1G, and S2A-C). This translates to a $>150-$ fold reduction in the bacterial load when compared to equivalent numbers of sporozoites obtained by SGD. Repeating the method with DAF produced sporozoites (dissected salivary glands used as input for FFE processing) demonstrated the successful removal of all detectable bacteria (Fig 11).

As an alternative to density gradients, we also tested whether rapid gel filtration with a Sephadex-based spin-column (5 min) could replace Accudenz, mirroring a method used with bovine sperm purification (51). Use of Sephadex resulted in sporozoite losses of $<10 \%$ compared with Accudenz, which resulted in sporozoite losses of up to $50 \%$. In parallel, we tested whether an interval zone electrophoresis (iZE) FFE method (see the Materials and Methods section) could add further improvements to our overall process (Fig S3A-D). Combining Sephadex with iZE, we were able to produce completely sterile sporozoites from whole mosquitoes (Fig S3D). However, because purity was associated with an additional substantial loss in yield ( $80 \%)$, the remainder of the development of the method (and experiments described below) used MAF purification and an FFE input of $100 \mathrm{mq} / \mathrm{ml}$ separated via CZE. Optimisation of Sephadex and iZE clearly has potential to further advancement of sterile sporozoite production at scale (important for good manufacturing process (GMP) licensure) and is the focus of future work.

\section{MAF sporozoites show improved in vitro infectivity compared with those from SGD}

Using the devised stepwise method described (MAF), we next sought to assess the in vitro infectivity of sporozoites isolated in this way. Sporozoite motility is often used as a primary indicator of sporozoite viability (23). Comparisons of the motility patterns of SGD $P$. berghei sporozoites versus those from MAF on a glass surface revealed no significant differences in the $2 \mathrm{D}$ motion patterns displayed (static, attached/waving or gliding) (52), either in terms of mean velocity or overall ratios of motion pattern (Fig 2A-C).

Extending infectivity analysis to in vitro infection of hepatoma or primary hepatocytes, RT-PCR analysis of $P$. berghei copy number, $24 \mathrm{~h}$ post infection (p.i.), showed a 1.5- and 2.1-fold increase in MAF sporozoite infectivity in HepG2 and primary rat hepatocytes when compared with that of SGD sporozoites, respectively (Fig 2D). Of note, the proportion of $P$. berghei exo-erythrocytic forms developing in primary rat hepatocytes $24 \mathrm{~h}$ p.i. was 13.5 -fold increased when MAF sporozoites were used rather than their SGD counterparts (infection rate of $5.4 \%$ versus $0.4 \%$ ) (Fig $2 \mathrm{E}$ ). At $52 \mathrm{~h}$ p.i., MAF sporozoites had completed maturation into late schizonts, as

conducted at $37^{\circ} \mathrm{C}, 17 \mathrm{~g}$, using mosquitoes blood-fed on uninfected mice $21 \mathrm{~d}$ before MAF extraction. (H) Bacterial growth (samples normalised to MEQ of $200 \mathrm{mq} / \mathrm{ml}$ ) at different stages from uninfected whole mosquito (M) origin purification. Samples were loaded onto the FFE machine at three different originating mosquito doses. (I) Bacterial growth (samples normalised to MEQ of $200 \mathrm{mq} / \mathrm{ml}$ ) at different stages from infected SGD-origin purification. Experiments show the mean of two technical replicates and error bars represent SEM. All treatments compared with dissected by unpaired two-tailed $t$ test using Bonferroni correction $\left(\mathrm{H}\right.$ : ${ }^{\star} P<0.01,{ }^{* \star} P<0.002$, ${ }^{\star \star *} P<$ $0.0002,{ }^{* * *} P<0.00002 ;$ I: $\left.{ }^{*} P<0.017,{ }^{* *} P<0.003,{ }^{* * *} P<0.0003,{ }^{* * * *} P<0.00003\right)$. 
A.
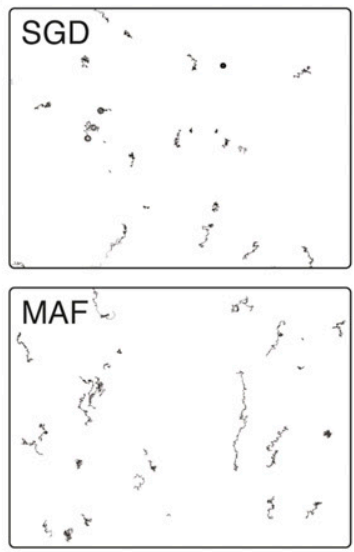

D.

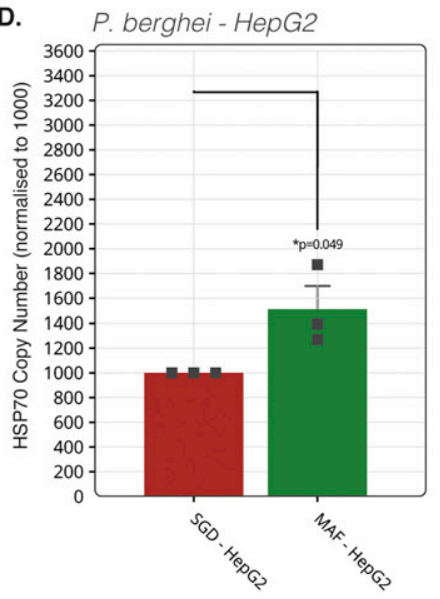

F.

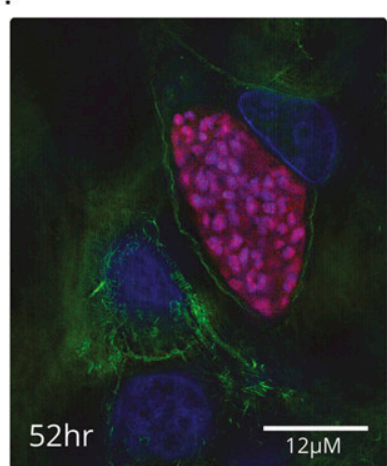

B.
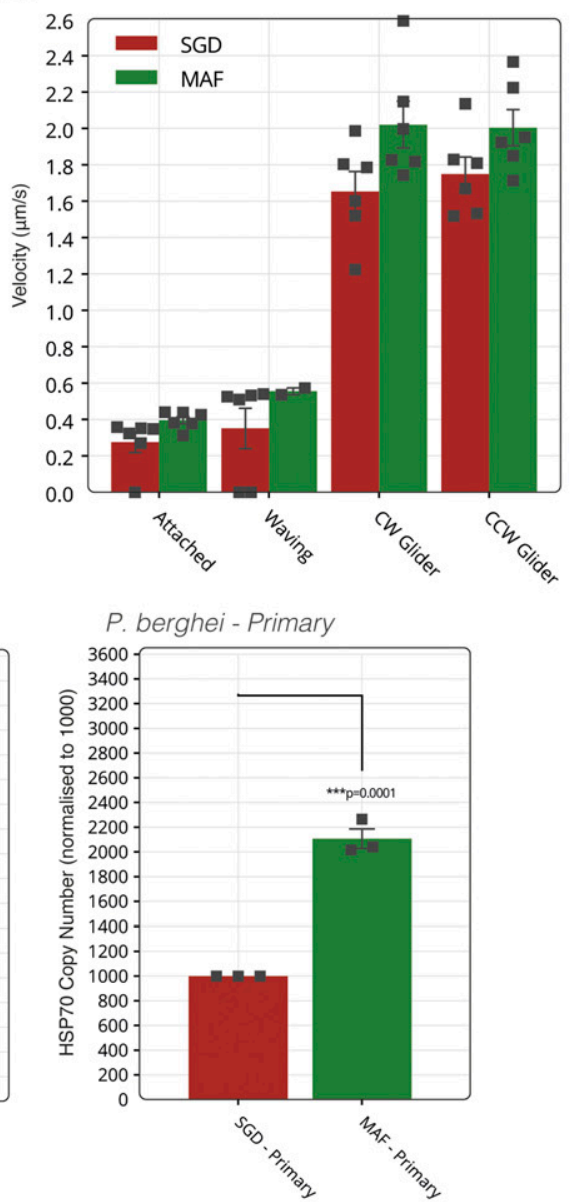

G. P. falciparum

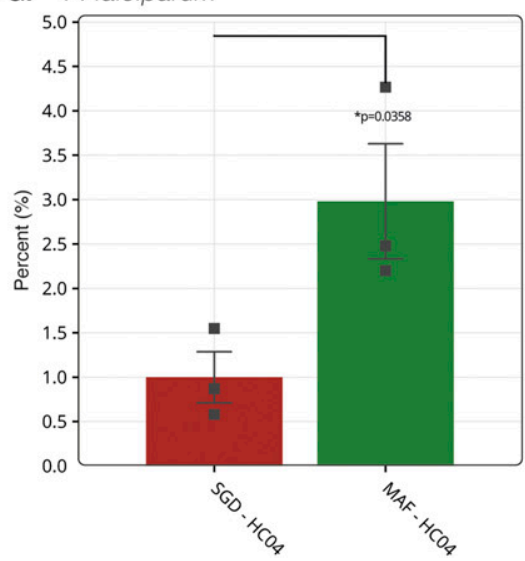

C.
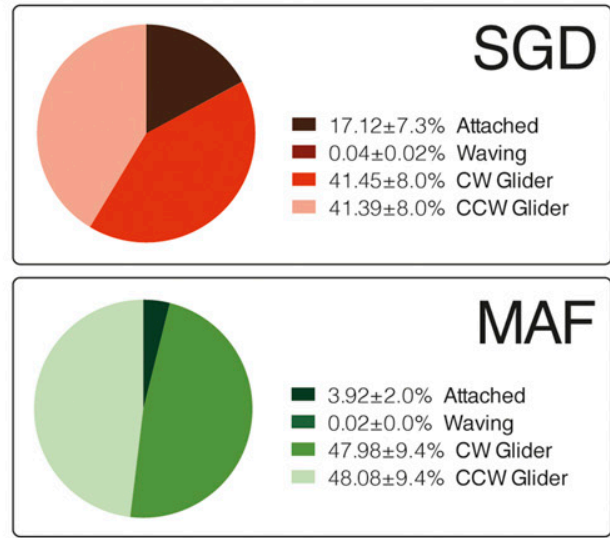

E.

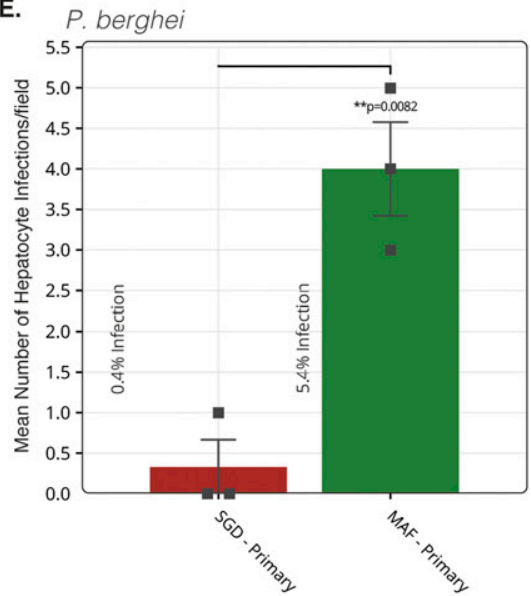

$\mathrm{H}$.

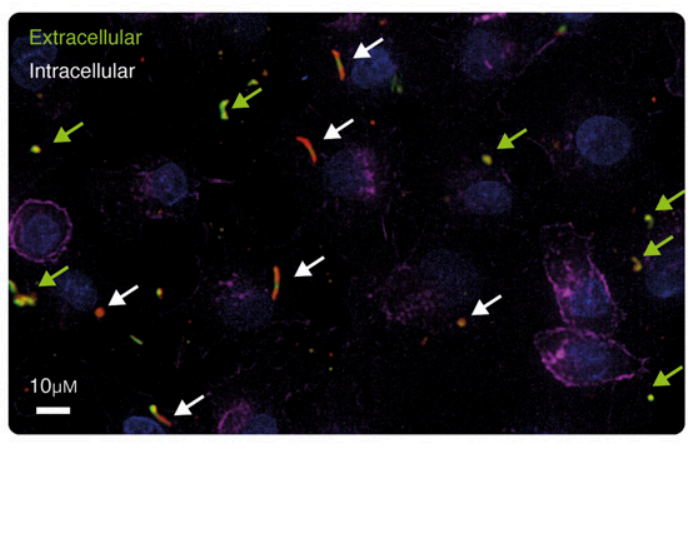

Figure 2. Assessment of purified sporozoite in vitro viability.

(A) Typical movement trails of MAF and salivary gland dissection (SGD) sporozoites over 600 frames at $2 \mathrm{~Hz}$. (B) Sporozoite gliding motility over $600 \mathrm{frames}$ at $2 \mathrm{~Hz}$ with a sliding nine frame average during each motility state over 600 frames at $2 \mathrm{~Hz}$. (C) Comparison of the percentage of all sporozoites in each state. Sporozoite tracking represents mean of two independent replicates and six technical replicates with groups compared using an unpaired two-tailed $t$ test. Bars represent means and error bars the SEM. A total of 10,672 and 8,370 sporozoites were counted for SGD and MAF, respectively. (D) Absolute RT-PCR quantification of parasite HSP70 housekeeping gene DNA copies normalised by host HSP60 gene in HepG2 (left) and primary rat (right) hepatocytes. Treatments for both HepG2 and primary hepatocytes were normalised to 1,000 hsp70 copies for the SGD treatment. Means of three independent replicates. (E) Mean counts of successful hepatocyte infections in primary rat hepatocytes measured by visual identification of six fields of view over 24-h time-lapse from three independent replicates. (F) Fluorescent image of late-stage schizont (52 h) captured using structured illumination microscope. Blue; nuclei, green; actin, red; mCherry parasite, pink; parasite actin (anti-5H3 [75]). (G) Means counts of initial hepatocyte invasions of Plasmodium falciparum sporozoites $4 \mathrm{~h}$ post infection in $\mathrm{HC}-04$ at a ratio of 1:5 cells to sporozoites. SGD treatment normalised to 1. Sporozoites stained for CSP to determine intracellular or extracellular location. One independent replicate with three technical replicates. (H) Immunoflourescent staining of HC-04 cells with fixed $4 \mathrm{~h}$ after infection with P. falciparum sporozoites and stained with anti-CSP (extracellular = green + red, intracellular = red only), DAPI for nuclear material (blue) and phalloidin for actin (purple). 
indicated by the presence of liver-stage merozoites (Fig 2F). These sporozoites also fully developed into late-stage exoerythrocytic schizonts when infected hepatocytes were extracted from rats and cultured ex vivo (Fig S4). Corroborating these results, assessment of MAF infected rat primary hepatocytes by flow cytometry, showed infection rates of $10.4 \%$ (302 of 2,808 cells, 1:1 ratio of cells to sporozoites) (Fig S5). Mirroring observations in P. berghei, humaninfective $P$. falciparum-derived sporozoites, processed through the same MAF process, were shown to have a threefold increased rate of invasion into HC-04 cells when compared to sporozoites isolated by SGD (Fig $2 \mathrm{G}$ and $\mathrm{H}$ ). The difficulties of setting up a robust human primary hepatocyte model for long-term in vitro development with $P$. falciparum precluded our ability to take these to late stage schizogony. Overall, analysis of P. berghei and P. falciparum sporozoites purified by our stepwise method nonetheless demonstrates that they exhibit at least equivalent and potentially superior infectivity in vitro.

\section{MAF sporozoites from segmented mosquitoes are more infectious than SGD in vivo}

As MAF sporozoites showed the potential for enhanced infectivity in vitro compared with SGD counterparts, further in vivo studies were performed to confirm this trend. Mice were inoculated with $P$. berghei sporozoites by i.v. injection and infectivity determined by measuring the time to reach $1 \%$ blood-stage parasitaemia (prepatent period), a standard measure of infectivity used in this field of research (53). Mice were inoculated i.v. with escalating doses of MAF purified $P$. berghei sporozoites, demonstrating that, independent of the inoculum size, MAF sporozoites were able to develop and establish a successful blood-stage infection (Fig 3A).

We next compared infectivity of MAF sporozoites with SGD sporozoites. Initially, 5,000 sporozoites obtained from either SGD, MA or MAF were given i.v. to mice with resulting blood-stage parasitaemia monitored. Partially purified MA sporozoites and fully purified MAF sporozoites showed a modest but significant delay in time to $1 \%$ parasitaemia compared to SGD (0.66 d longer for MA, ${ }^{* *} P$ $=0.0049 ; 0.59 \mathrm{~d}$ longer for MAF, ${ }^{* \star} P=0.0031$; Mantel-Cox Test) (Fig $3 \mathrm{~B})$. This result was in contrast to in vitro infections, which were significantly increased with MAF sporozoites. It is clear that sporozoites purified from whole mosquitoes will necessarily include a proportion of immature haemocoel sporozoites that have yet to reach the salivary glands. We therefore reasoned that this reduction in in vivo infectivity could be due to the presence of less infective sporozoites in the MA and MAF inoculum. Indeed, whereas sporozoites obtained from the mosquito haemocoel can infect
A.

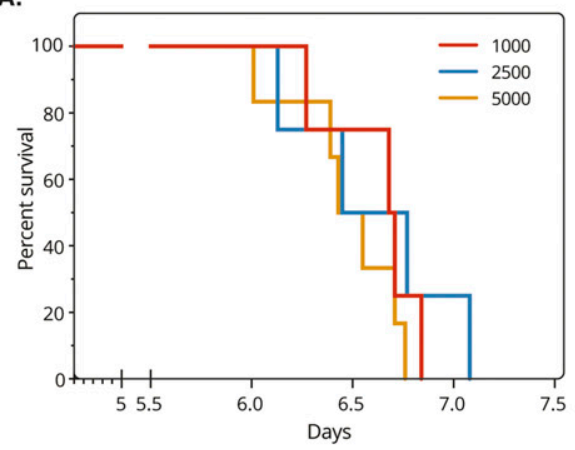

D.

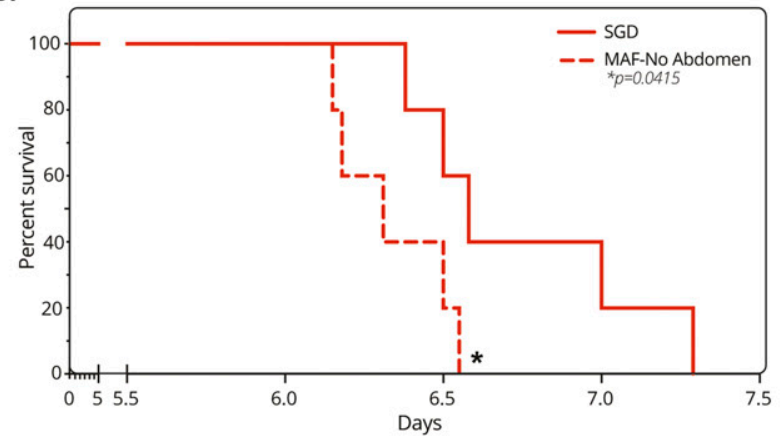

B.

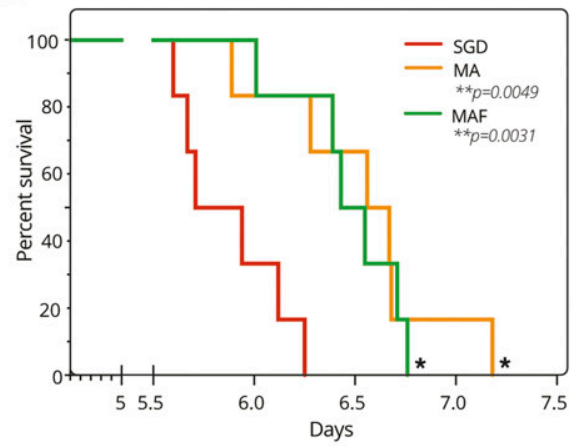

c.

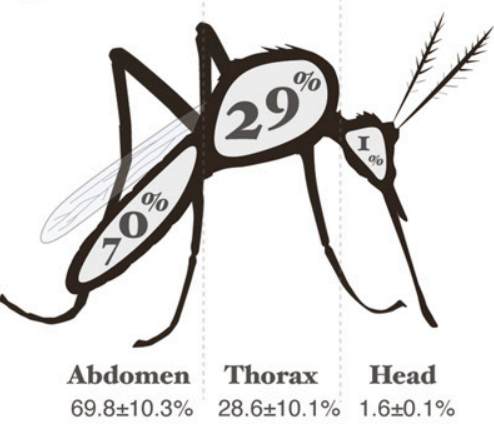

E.

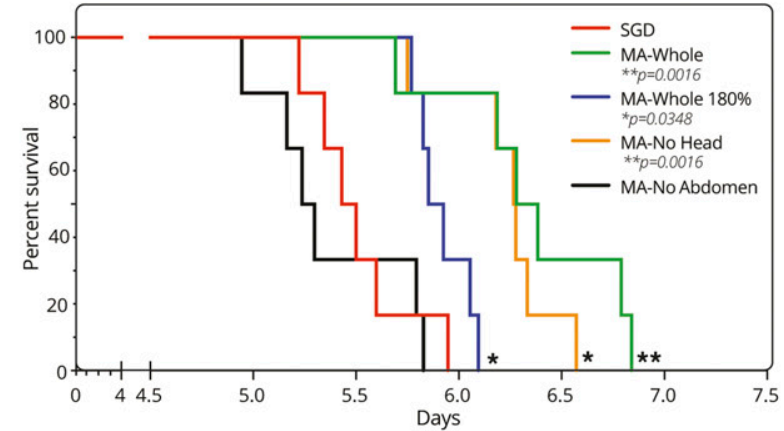

Figure 3. Assessment of purified sporozoite in vivo viability.

(A) Kaplan-Meier survival curve of mice challenged i.v. with increasing doses of sporozoites from MAF. Six mice per group. End point classed as $1 \%$ parasitaemia. (B) Kaplan-Meier survival curve of mice challenged i.v. with 5,000 sporozoites from different purification steps. Six mice per group. End point classed as $1 \%$ parasitaemia, treatments compared by Mantel-Cox statistical test. (C) Sporozoite distribution of infected mosquitoes, average from 85 mosquitoes, two experimental replicates. Values show mean with SEM. Raw sporozoite numbers per mosquito are as follows: abdomen: 95,950; thorax: 42,500; head: 2,292. (D) Kaplan-Meier survival curve of mice challenged i.v. with 1,000 sporozoites from MAF-No Abdomens purified (MAF from mosquitoes with abdomens removed before homogenisation) and salivary gland dissection origin. Six mice per group. End point classed as 1\% parasitaemia, treatments compared by Mantel-Cox statistical test. (E) Kaplan-Meier survival curve of mice challenged with 5,000 sporozoites obtained by MA purification from different mosquito sources or salivary gland dissection origin. Six mice per group. Death classed as $1 \%$ parasitaemia, treatments compared by Mantel-Cox statistical test. 
hepatocytes in vitro and in vivo (30), studies with salivary gland sporozoites have generally exhibited markedly increased in vivo virulence compared to less mature haemocoel sporozoites $(30,47)$. To investigate the relative proportion of immature midgut-derived/ haemocoel sporozoites in whole mosquito homogenates, mosquitoes at $21 \mathrm{~d}$ post infectious bloodmeal were therefore separated into abdomen, thorax (containing the salivary glands) and head. Segmentation of the mosquito in this manner revealed that a significant number of sporozoites are found in the abdomen (70\%) compared to the thorax (29\%), which contains the salivary glands (Fig 3C).

To address whether inclusion of haemocoel sporozoites was responsible for reduced infectivity, we subtracted immature sporozoites from the initial inoculum by removing mosquito abdomens to assess whether this reverted the delay in time to patency. Mosquito abdomens were removed before the initial homogenisation step in our purification platform (MAF-No Abdomen) and, as a measure of sporozoite in vivo infectivity, the time to $1 \%$ blood parasitaemia was monitored as before. Notably, in mice infected with MAF-No Abdomen sporozoites there was now a marked increase in infectivity (time to $1 \%$ parasitaemia) when compared with SGD sporozoites ( ${ }^{\star} P=0.04$; Mantel-Cox Test; Fig 3D). Of note, this increase in infectivity was only seen when sporozoites went through all steps of the purification pipeline, as no differences were observed between mice challenged with SGD and partially purified MA-No Abdomen sporozoites (MA with abdomens removed before homogenisation) (Fig 3E). This indicates that the FFE stage of the method is key to increasing sporozoite infectivity, while also showing that by increasing the dose of sporozoites extracted from whole mosquitoes with intact abdomens (i.e., accounting for the immature population in the haemocoel) it is possible to compensate for this delay (180\%, 1.8-fold increase; Fig 3E). These data show that the purification method described can yield sporozoites with increased in vitro and in vivo infectivity; however, to observe this increase in infectivity directly in vivo, dose must be compensated for to account for immature haemocoel-derived sporozoites.

\section{Vaccination with irradiated MAF sporozoites confers sterile protection}

Having developed a process that produces sporozoites with high purity and high infectivity, we next sought to assess the potential of MAF sporozoites as a radiation-attenuated sporozoite vaccine (RASv). To enable an effective comparison of efficacy with sporozoites of the same maturity to those from SGD (i.e., to account for immature haemocoel sporozoites for fair head-to-head comparison), we used MAF sporozoites from mosquitoes without abdomens for immunisation experiments. Before immunisation, the effective irradiation dose was determined to be 60 Gy by i.v. inoculation with varying doses of $y$-irradiated sporozoites (Fig 4A). Mice were immunised i.v. using a three-immunisation regime of 40,000 irradiated sporozoites, 2 wk apart. In parallel, cohorts of control mice were immunised with plain medium as controls. Immunisation efficacy was assessed by challenging with five infectious mosquito bites (Fig 4B) (46). Immunisation with P. berghei (wild-type PbANKA) or $P$. falciparum (wild-type NF54) MAF-RASv sporozoites i.v. achieved complete protection against native $P$. berghei or chimeric $P$. berghei expressing $P$. falciparum CSP (PbANKA-PfCSP), respectively (Fig $4 \mathrm{C}$ and D). The level of protection (sterile protection) was comparable to that offered by SGD-RASv. Equivalent total IgGs measured against whole sporozoites pre-challenge were found in the serum from immunised animals irrespective of the source of $P$. berghei sporozoites (Fig 4E).

Finally, considering the practical development and utilisation of a whole sporozoite vaccine and the comparatively challenging nature of i.v. administration, we sought to address whether alternative routes of immunisation such as intramuscular (i.m.) might become possible given the shortened time to patency of MAF-No abdomen produced parasites. Previous attempts at i.m. immunisation using $P$. berghei demonstrated a level of protection around $30 \%$ (47). Intramuscular immunisation of 40,000 irradiated MAF sporozoites with two boosts was given with the commercial adjuvant AddaVax, a squalene-based oil-in-water nano-emulsion (InvivoGen). Sporozoites produced by our MAF method showed $70 \%$ and $67 \%$ protective efficacy for both $P$. berghei and $P$. falciparum MAF-RASv immunisation compared to non-immunized controls, respectively. Comparison of total IgG against sporozoites between i.v. and i.m revealed similar antibody titres for $P$. berghei immunisations (Fig 4E), whereas a modest increase in titre was observed with $P$. falciparum between i.m. and i.v. routes of immunisation (Fig 4F). Although direct comparison with data under different conditions from previous studies is challenging (47), it is clear that MAFproduced sporozoites show great potential for whole-sporozoite vaccination, which critically is not dependent on manual SGD.

\section{Discussion}

We present here a robust stepwise method for the isolation of large quantities of pure malaria sporozoites that does not require manual SGD. Sporozoites isolated by this dissection-independent method exhibited improved sterility and enhanced infectivity in vitro when compared to SGD sporozoites, improved in vivo infectivity when compared with sporozoites of the same maturity (MAF without abdomens), and conferred sterile protection in a mouse challenge model. With demonstrated application to both rodent $P$. berghei and human-infective $P$. falciparum, sporozoite production using this process represents a potentially transformative technology that has multitude applications. Not least, this stepwise process can serve as an optimal starting point for development of a dissection-independent manufacturing process for GMP grade whole-sporozoite vaccines.

By combining bulk mosquito homogenisation, Sephadex filtration (or density centrifugation), and FFE separation (abbreviated to MAF), sporozoites purified using the stepwise method developed here could be obtained significantly faster than SGD and with all detectable mosquito-associated protein removed. In addition to the improved levels of purity gained, MAF sporozoites also showed a markedly improved infectivity in vitro for both rodent and human malaria parasites. The reduced overall time required and consistency of production are likely to be key factors in determining in vitro infectivity. However, several additional factors likely account 
A.

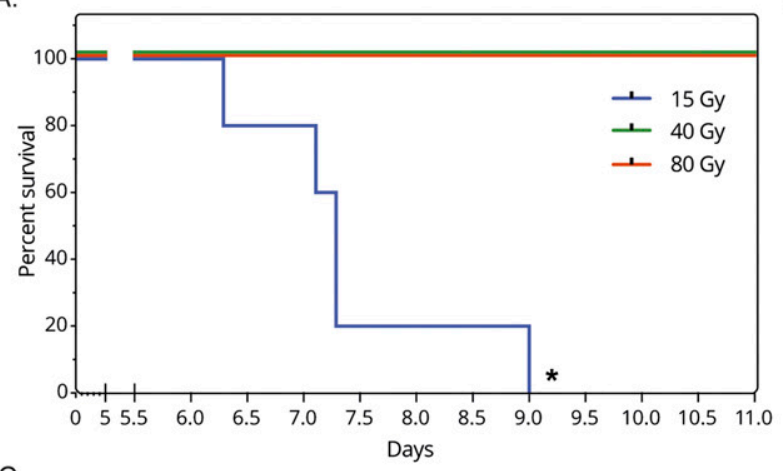

C.

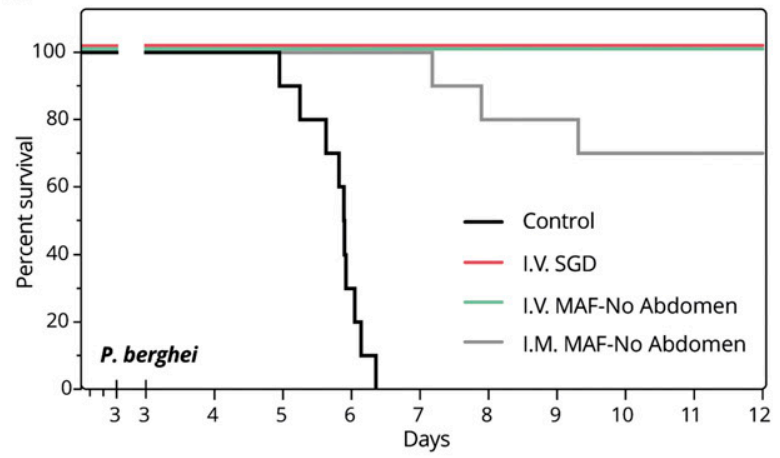

B.

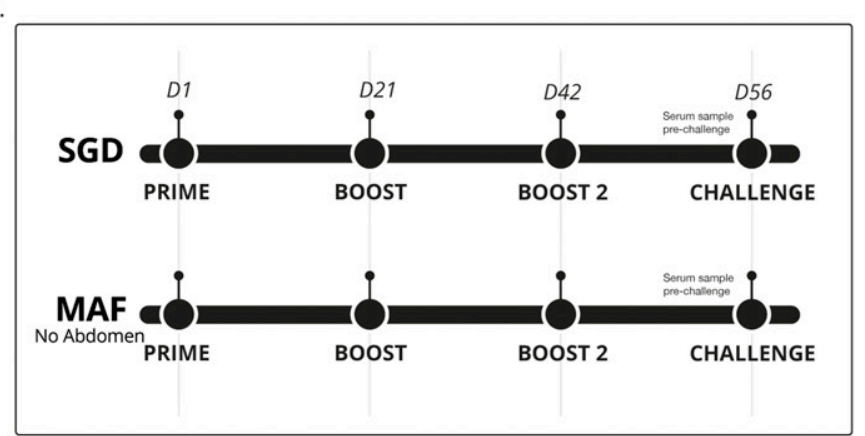

D.

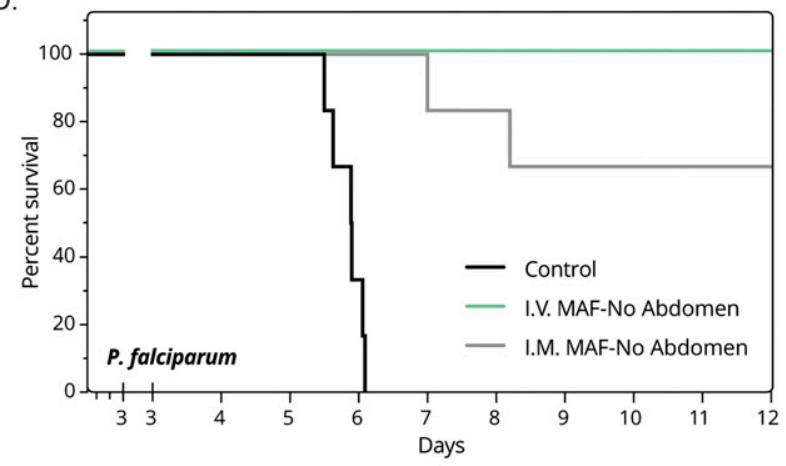

E.

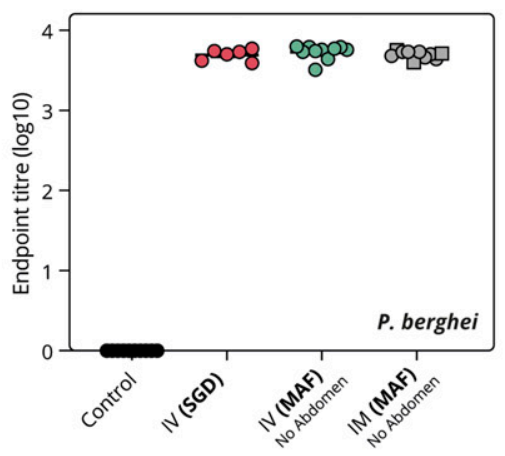

F.

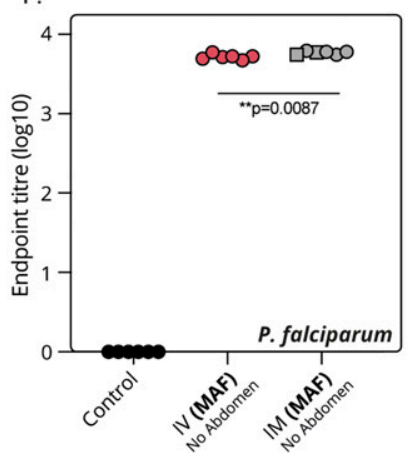

Figure 4. Purified sporozoites as a viable vaccine.

(A) Kaplan-Meier survival curve of mice challenged i.v. with 1,000 Plasmodium berghei sporozoites from MAF-No abdomen purified and gamma irradiated. Four mice per group. End point classed as 1\% parasitaemia. (B) Schematic of vaccination regime used. Sporozoites were either from salivary gland dissection or MAF-No Abdomen origin, then gamma irradiated. (C) Immunisation i.v. or i.m. of Balb/c mice with irradiated P. berghei sporozoites from either manual salivary gland (salivary gland dissection) dissection or MAF-No Abdomen. Mice given three immunisations of 40,000 sporozoites, 2 wk apart followed by challenge with five infectious mosquito bites. Ten mice per group. End point classed as 1\% parasitaemia. (D) Immunisation i.v. or i.m. of Balb/c mice with irradiated Plasmodium falciparum sporozoites from MAF-No Abdomen. Mice given three immunisations of 40,000 sporozoites, 2 wk apart followed by challenge with five infectious mosquito bites. Six mice per group. End point classed as $1 \%$ parasitaemia. (E) Total titres of IgG antibodies against P. berghei sporozoite lysate in mouse serum before challenge (F) Total titres of IgG antibodies against $P$. falciparum sporozoite lysate in mouse serum before challenge. Squares indicate mice not protected.

for this improvement in infectivity. When MAF and SGD sporozoites were added to primary human hepatocytes, SGD treatment was found to be associated with abnormal human cell morphology and reduced cell numbers (Fig S6), suggesting mosquito contaminants may be detrimental to host-cell growth, reducing overall viability of hepatocytes. To account for this confounding variable seen with in vitro experiments, in vivo infection studies exploring whether our purified sporozoites directly exhibited improved infectivity compared with SGD sporozoites were undertaken with MAF sporozoites of comparable maturity (i.e., deriving from mosquitoes with abdomens removed). These sporozoites of comparable maturity showed improved infectiousness, suggesting that overall our process likely contributes not just to purity but also to improved infectiousness in vitro.

When exploring other possible reasons for improved infectivity we noted that MAF sporozoites had 4.3 times more cleaved CSP on their surface than SGD sporozoites (Fig S1D) $(48,49)$. Previous work has explored the importance of CSP processing on $P$. berghei sporozoite invasion. Of note, genetically altered sporozoites expressing a precleaved CSP showed greater levels of in vitro infectivity (27). The proportion of CSP that is processed following MAF purification appears to be accelerated and therefore may be a contributing factor to improved infectivity of sporozoites isolated in this way, although this 
will require further experimental validation. Finally, a recent study identified the mosquito salivary protein, mosGILT as negatively modulating sporozoite motility (25). Other mosquito-associated factors that reduce hepatocyte infectivity may be forthcoming and would likely be purified away from sporozoites using the MAF protocol, which may further contribute to improved hepatocyte infectivity. Controlling these factors in the future may help advance in vitro liver-stage systems and high-throughput assays that yield relatively low numbers of infected hepatocytes after exposure to $P$. falciparum sporozoites $(20,54,55)$. Ultimately, this should advance the development of comparable liver-stage platforms to those available for asexual blood-stage or sexual stage high throughput in vitro platforms (56). Further work is clearly warranted towards this.

Although MAF sporozoites showed the same motility patterns as SGD, this was somewhat unexpected given that MAF sporozoites originate from the entire mosquito. Sporozoites originating from less mature stages, such as those in oocysts or the haemolymph, are typified by reduced gliding motility compared to mature salivary-gland resident sporozoites $(30,47,52)$. Indeed, we found that $70 \%$ of sporozoites in a typical day 21 post mosquito feed were abdominal in origin, conforming with previous observations (27) and indicating that most MAF sporozoites would be derived from less mature oocyst or haemolymph stages. Studies on the infectiousness of sporozoite developmental stages have shown that oocyst sporozoites (i.e., within the abdominal section) are more than 1,000-fold less infectious than salivary gland sporozoites by i.v. challenge (57). Thus, a significant proportion of injected sporozoites post-isolation may potentially be poorly infective, supporting a long-held belief that gliding per se may not be a good indicator of infectiousness. Our own infection data corroborate this, showing that use of whole mosquito homogenate was associated with a delay in blood-stage parasitaemia when compared with production via our process but with prior removal of the abdomen. Removal of the abdomen reverted any delay seen and demonstrated an increased time to patency compared to SGD (Fig 3D). Because removal of abdomens but without FFE (MA) did not advance infectivity (Fig 3E), this indicates that it is the FFE step that is critical to improving the viability of MAF sporozoites over SGD and not the removal of the abdomen on its own. Thus, efforts seeking to enrich specifically for highly infectious sporozoites (though not necessarily relevant for vaccination) may require prior abdomen removal from the mosquito before FFE for maximal infectivity. We note, however, that infectivity per se may not be the only requirement for overall efficacy in vaccination, indeed having a mixed population of immature sporozoites with those that are hyper-infective may be beneficial for broadly activating different arms of the immune system.

Sterile protection in mice is possible after administration of SGD sporozoites. Mirroring many similar studies, once irradiated, our MAF sporozoites showed full protection in both rodent and human challenge models via the i.v. immunisation route with similar antibody titres. Because it has recently been demonstrated that there are mosquito associated proteins that are able to modify the human immune response, it may be expected that such proteins could also interfere with the efficacy of a whole-sporozoite vaccines. Indeed, in agreement with this, recent work (58) showed that using Accudenz with SGD sporozoites to reduce total protein load was associated with improved pre-primed sporozoite (boost) specific CD8 T-cell responses compared with standard SGD. Thus, mosquito contaminants may cause innate immune up-regulation in vivo with unknown, if not confounding, effects on vaccination studies. Because MAF sporozoites showed a marked reduction in mosquito-associated protein and bacterial load, with potential for sterility using the iZE FFE method, this suggests that MAF sporozoites may well outperform SGD sporozoites in future head-to-head immunogenicity comparisons. Our ability to gain significant protection by i.m. immunisation route (60-70\%) is certainly suggestive of markedly improved immunogenicity than previously obtained with SGD alone (47). Interestingly, recent work has shown intradermal immunisation with SGD-RASv was also associated with reduced efficacy compared with i.v. when challenged intradermally (59). Further work testing doses of sporozoites between MAF and SGD will be required to accurately assess comparative immunogenicity of different sporozoite sources and routes of immunisation.

In conclusion, the work presented here shows the development of a complete stepwise method for purification of large numbers of highly infectious sporozoites in a scalable format that is entirely compatible with basic biological, drug-screening, and whole-parasite vaccine studies. Our process yields sporozoites at higher purity compared with those from dissected preparations alone and is associated with a marked increase in in vitro hepatocyte infections and, once immature sporozoites from the abdomen are controlled for, enhanced in vivo infectivity. Sporozoites harvested by our process show markedly reduced levels of contaminants, can be produced aseptically and, critically, can be used to demonstrate high protective efficacy after both i.v. and intramuscular immunisation. For basic sciences, this stepwise method will be an important step towards single cell -omic studies that require large amounts of highly pure sporozoites, free from mosquito-associated contaminants that will unavoidably limit some of the scope or depth of coverage of such studies $(60,61)$. Concurrently, application and future adaptation of this technology to a GMP-compliant vaccine development pipeline, including genetically attenuated sporozoites $(62,63)$, offers the tantalizing opportunity to develop and manufacture pure, viable, immunogenic whole-parasite sporozoite vaccines at a dramatically increased scale when compared with current methods. Production of sporozoites at scale will no doubt advance our understanding of malaria liver-stage biology and help address the critical global need of an effective antimalarial vaccine.

\section{Materials and Methods}

\section{Mosquito maintenance}

Anopheles stephensi mosquitos used for experiments were raised at $28^{\circ} \mathrm{C}, 70 \%$ relative humidity with a 12 -h light cycle. Larvae were fed with fish pellets and adults maintained on $10 \%$ fructose (reared by Alex Fyfe and Mark Tunnicliff).

\section{$P$. berghei maintenance and infection}

Two transgenic P. berghei ANKA lines were used in this study that express either mCherry or GFP under control of the uis4 promoter. 
This promoter drives transgene expression specifically in sporozoites and liver stages. The transgene expression cassettes of both lines have been introduced into the neutral p230p gene locus by the method of gene insertion/marker out (GIMO) transfection (64). The generation and characterisation of the mCherry-expressing line mCherry@Pbuis4_230p (line 2204) has been described previously (65). The generation and characterisation of the GFP-expressing line GFP @Pbuis4_230p (line 2227) was generated as follows: The P. berghei ANKA line GIMO parent line 1596cl1 (64) was used for transfection with a construct (pL1962) which targets the neutral p230p locus (PBANKA_030600) and inserts GFP::Luciferase expression cassette, thereby removing the selectable marker (SM) consisting of human dihydrofolate reductase and the yeast cytosine deaminase and uridyl phosphoribosyl transferase (hdhfr:: $y f(u)$, according to the GIMO transfection technique, which has previously been described (64). The transfection vector, which lacks a drug SM cassette, was obtained using the using the standard GIMO DNA construct pL0043 (64). The expression cassette contained the GFP::Luciferase flanked by the $5^{\prime}$ and $3^{\prime}$ promoter and transcription terminator sequences of $P$. berghei uis 4 gene (PBANKA_0501200), which were amplified from P. berghei ANKA wild-type genomic DNA. The regulatory sequences of uis 4 gene were chosen to express GFP::Luciferase in sporozoites and liver stages $(66,67)$. Sequences of primers used for pL1962 construct generation are listed in Table S2. Transfection (exp. 2227) of 1596cl1 was performed using standard transfection methods (68) and negative selection was applied by treating mice with the 5-fluorocytosine (5-FC) in drinking water as described for GIMO transfection (64). The selected parasites were cloned by limiting dilution in mice and line 2227cl6 was further characterized for correct integration of GFP::Luciferase expression cassette into the p230p locus by diagnostic PCR and Southern analysis of pulsed-field gel electrophoresis-separated chromosomes (68). Sequences of primers used for PCR genotyping are listed in Table S3. The selected parasite 2227cl6, named GFP::Luc@Pbuis4_230p, contains the fusion gene gfp-luciferase under the control of the uis 4 regulatory sequences integrated into the neutral p230p locus and is SM free (see Fig S7A and B and source data). For vaccination studies mice were immunised with either the PbANKA 2.34 (wild type) or NF54 (wild type). Subsequent challenge was with either PbANKA 2.34 or PbANAKA 2.34 transgenic for P. falciparum CSP (PbANKA-PfCSP chimeric [69]).

For infection of mice, cryopreserved parasitized RBCs (day 5) were thawed and injected into naive Balb/c mice by the intraperitoneal (i.p.) route and An. stephensi mosquitos allowed to feed on anesthetised mice with 1-2\% blood-stage parasitaemia. 7-10 d later, these mosquitoes were allowed to take an additional bloodmeal on naive Balb/c mice to increase sporozoite yields. Blood-fed mosquitos were maintained at $19^{\circ} \mathrm{C}$ at $70 \%$ relative humidity for 19-22 d before sporozoites were extracted. Infection yielded an average salivary gland load of 40,000 sporozoites per mosquito.

\section{P. falciparum maintenance and infection}

The wild-type NF54 P. falciparum strain was used in this study and cultured in vitro and gametocytes induced as per Delves et al (70).
Briefly, asexual cultures were grown in RPMI 1460, supplemented with $25 \mathrm{mM}$ Hepes (Life Technologies), $50 \mu \mathrm{g} \mathrm{l^{-1 }}$ hypoxanthine (Sigma-Aldrich) and 10\% A+ human serum (Interstate Blood-Bank). Gametocyte cultures were grown in RPMI 1640 supplemented with $25 \mathrm{mM}$ Hepes (Life Technologies), $50 \mu \mathrm{g} \mathrm{l^{-1 }}$ hypoxanthine (SigmaAldrich), $2 \mathrm{~g} \mathrm{l}^{-1}$ sodium bicarbonate (Sigma-Aldrich), 5\% A+ human serum (Interstate Blood-Bank), and 0.5\% AlbuMAX II (Life Technologies). For standard membrane-feeding assays, 15-17 d-old gametocyte cultures were diluted in fresh RBCs and human serum at $50 \%$ haematocrit and used to feed female overnight-starved mosquitoes. Mosquitoes were maintained for 16-18 d before sporozoites were extracted. Infection yielded an average salivary gland load of 20,000 sporozoites per mosquito.

\section{Manual salivary gland dissection}

Mosquitoes were sedated on ice for $10 \mathrm{~min}$, then placed on a glass slide with $100 \mu \mathrm{l}$ complete Schneider's Drosophila medium (1\% FBS, $4^{\circ} \mathrm{C}, \mathrm{NaHCO}_{3}$ free, Pan-Biotech) and whole salivary glands removed by gentle separation of the head using micro-forceps. Both sets of glands were gently cleaned to remove other tissues and then placed into a glass Dounce tissue grinder on ice using $2 \mu \mathrm{l}$ fresh medium. The glass slide was cleaned between each dissection. Each dissection took 45-90 s and was carried out for no more than 2-3 h maximum to reduce loss of infectivity. To release sporozoites the salivary glands were homogenised with three gentle but firm grinds using the pestle. The sample was transferred to protein lobind tubes (Eppendorf) used to prevent loss of sporozoites by adhesion to plastic-ware and mixed well before a sample was added to a haemocytometer and the average of four 16 square fields counted. Sample was diluted if too concentrated to accurately count.

\section{Homogenisation and accudenz gradient purification/Sephadex}

Mosquitoes sedated on ice were placed in a Petri dish with $2 \mathrm{ml}$ (per 400 mosquitoes) complete Schneider's Drosophila media and gently homogenised with the end of a 10-ml syringe barrel for 30-60 s or using a gentleMACS homogeniser (Miltenyl Biotech). Liquid was removed and passed through a $100-\mu \mathrm{M}$ cell strainer in a $50-\mathrm{ml}$ centrifuge tube. A further $1.5 \mathrm{ml}$ media was added to the petri dish, gently homogenised and passed through the $100-\mu \mathrm{M}$ filter. This was repeated twice more but with more vigorous grinding. Finally, the filter was washed with $1 \mathrm{ml}$ media. The filtrate was subsequently passed through a 70-, 40-, and 20- $\mu \mathrm{M}$ filter and each washed with $1 \mathrm{ml}$ media (M). All steps were carried out on ice. On some occasions, the head and/or abdomens were removed before homogenisation. $1 \mathrm{ml}$ homogenate was loaded onto a $3-\mathrm{ml}$ Accudenz cushion $\left(4^{\circ} \mathrm{C}\right)$ in a $15-\mathrm{ml}$ centrifuge tube and centrifuged $\left(2,500 \mathrm{~g}, 4^{\circ} \mathrm{C}\right)$ as per reference 24 .

Subsequently, $400 \mu \mathrm{l}$ was taken from the sporozoite enriched boundary (at the $3 \mathrm{ml}$ mark). $1 \mathrm{ml}$ aliquots of Accudenz sporozoites were put into 2-ml protein lo-bind tubes (Eppendorf), made up to $2 \mathrm{ml}$ with complete Schneider's media and centrifuged $(12,000 \mathrm{~g}$, $4^{\circ} \mathrm{C}, 3 \mathrm{~min}$ ). The resultant pellet was re-suspended in complete Schneider's media (MA). If the sample was to be used for FFE it was re-suspended to a mosquito equivalent (ME) of $200 \mathrm{mq} / \mathrm{ml}$ 
(mosquitoes/ml; unless stated otherwise), which was based on the original number of whole mosquitoes homogenised and the final volume this was in. The resultant sporozoite suspension was in most cases further purified by FFE.

Alternatively, SG-15 Sephadex medium was prepared in 3\% sodium citrate at a 1:1 vol/vol ratio overnight. Subsequently a PD10 column was packed with $5 \mathrm{~cm}$ depth Sephadex and homogenate applied. This was centrifuged for 1-5 min at 46-413g. Eluted samples were subsequently applied to FFE.

\section{FFE purification}

Before sporozoite extraction, the FFE machine (FFE Service GmbH) was setup for CZE using a 0.5-mm ZE spacer. A separation buffer of $10 \mathrm{mM}$ triethanolamine (TEA), $10 \mathrm{mM}$ glacial acetic acid (HAC), and $250 \mathrm{mM}$ sucrose was used with a stabilisation buffer of $100 \mathrm{mM}$ TEA, $100 \mathrm{mM} \mathrm{HAc}$, and $250 \mathrm{mM}$ sucrose injected into the separation chamber at $150 / 300 \mathrm{ml} / \mathrm{h}$. Electrodes were kept in $100 \mathrm{mM}$ TEA, $100 \mathrm{mM} \mathrm{HAc}$, and $250 \mathrm{mM}$ sucrose with a voltage of 900/750 $\mathrm{V}$ and current and power limit of $150 \mathrm{~mA}$ and $150 \mathrm{~W}$, respectively. MA sample was mixed 1:1 with separation buffer (now at $100 \mathrm{mq} / \mathrm{ml}$ ) and injected into the separation chamber at the cathode end at a rate of 1,600 $\mu \mathrm{l} / \mathrm{hr}$ and fractions collected $14 \mathrm{~min}$ after injection started and stopped $14 \mathrm{~min}$ after sample finished. Fractions were collected at $4^{\circ} \mathrm{C}$ in $2 \mathrm{ml}$ protein lo-bind deep-well plates (Eppendorf) containing $400 \mu \mathrm{L}$ complete Schneider's medium. The peak sporozoite fraction(s) was identified by a haemocytometer and centrifuged in $2 \mathrm{ml}$ protein lo-bind tubes ( $\max , 4^{\circ} \mathrm{C}, 3 \mathrm{~min}$ ) and the pellet re-suspended in 100-500 $\mu$ l complete Schneider's media (MAF). To compare purification stages all samples were resuspended to the same ME. FFE ME dose was calculated based on the volume collected in the peak fraction. Alternatively, the FFE machine was setup for iZE using a 0.2- $\mathrm{mm}$ spacer with identical separation buffers. Settings were 1,200 V, $150 \mathrm{~mA}$, and $120 \mathrm{~W}$ with injection at 2,000 $\mu \mathrm{l} / \mathrm{h}$.

\section{Hepatocyte culture}

Tissue culture plates (MatTek Corporation) were pre-coated overnight or using plasma-treatment with a $0.1 \mathrm{M}$ bicarbonate buffer (pH9.4) (71) of collagen I, collagen IV, fibronectin, and laminin ( $1 \mu \mathrm{g} / \mathrm{cm}^{2}$; Sigma-Aldrich). Human HepG2 hepatoma cell lines were maintained in complete DMEM (10\% FBS, $1 \%$ penicillin/ streptomycin, $5 \%$ L-glutamine; Sigma-Aldrich) at $37^{\circ} \mathrm{C}$ with $5 \%$ $\mathrm{CO}_{2}$. A confluent monolayer was maintained using an $18 \mathrm{G}$ syringe needle. HC-04 cells were maintained in complete medium (DMEM supplemented with F12, 10\% FBS, 1\% penicillin/streptomycin, and 5\% L-glutamine; Sigma-Aldrich). To obtain primary hepatocytes, male Wistar rats (Crl:CD(SD), strain 001) were anesthetised and a 21G cannula was inserted into the hepatic portal vein and secured/ sealed using tissue adhesive (3M). Liver perfusion medium $\left(37^{\circ} \mathrm{C}\right.$; Thermo Fisher Scientific) was pumped through the cannula at $10 \mathrm{ml} / \mathrm{min}$ using a peristaltic pump and once the liver started to lighten (within $30 \mathrm{~s}$ ) the speed was adjusted to $20 \mathrm{ml} / \mathrm{min}$. Subsequently the inferior vena cava was cut and over the next 5 min blocked two to three times and the pump increased to $40 \mathrm{ml} / \mathrm{min}$. After successful perfusion, the media was exchanged for liver digest medium $\left(37^{\circ} \mathrm{C}\right.$; Thermo Fisher Scientific) and the same blocking procedure carried out for $8 \mathrm{~min}$. The liver was subsequently transferred quickly to $4^{\circ} \mathrm{C}$ complete DMEM on ice, the liver disrupted (using gentleMACS homogeniser), and the passed through $100-\mu \mathrm{M}$ cell strainers. The cell suspension was washed twice $(50 \mathrm{~g}$, $5 \mathrm{~min}, 4^{\circ} \mathrm{C}$ ) with a final re-suspension into $19 \mathrm{ml}$ complete DMEM and $20 \mathrm{ml}$ sterile isotonic percoll (90\% percoll, 10\% 10× PBS) and centrifuged $\left(1.06 \mathrm{~g} / \mathrm{ml}, 100 \mathrm{~g}, 10 \mathrm{~min}, 4^{\circ} \mathrm{C}\right)$ to remove debris and dead cells (percoll purification modified from reference 72 ). The pellet was washed in complete DMEM and used to seed plates. Importantly the plates were not moved for 30 min to allow the cells to adhere evenly across the plate. They were then transferred to an incubator $\left(37^{\circ} \mathrm{C}, 5 \% \mathrm{CO}_{2}\right)$ for $1-2 \mathrm{~h}$ before medium was exchanged with serum-free hepatocyte growth medium (Promocell) which was exchanged every 12-15 h.

\section{Sporozoite motility assessment}

Sporozoites were added to $37^{\circ} \mathrm{C}$ complete DMEM and centrifuged $(413 \mathrm{~g}, 4 \mathrm{~min})$ in glass bottom tissue culture plates for sporozoites to sediment. Fluorescent images were captured at $2 \mathrm{~Hz}$ for 600 frames at 20x magnification. Motility was assessed using the ToAST ImageJ plugin (52).

\section{Rodent sporozoite challenge}

P. berghei sporozoites were extracted from infected mosquitoes using one of the described methods and diluted in complete Schneider's Drosophila medium ( $\left.1 \% \mathrm{FBS}, 4^{\circ} \mathrm{C}\right)$. Mice were placed in a $37^{\circ} \mathrm{C}$ heat-box for $10 \mathrm{~min}$ before injection of $50 \mu \mathrm{l}$ i.v. into either lateral tail vein of restrained mice. From day 4-5, parasitaemia was monitored by Giemsa-stained thin-blood film until $3 \mathrm{~d}$ of positive smears were obtained, mice were then euthanized. 1,000 red blood cells were then counted from each slide over multiple fields of view to determine parasitaemia. Treatments were blinded to the user. Time to $1 \%$ parasitaemia was then calculated by linear regression. If parasites were not detected by day 14 the mice were euthanized.

\section{Rodent vaccination}

Before immunisations sporozoites were diluted in Schneider's Drosophila media to $80 \times 10^{4} / \mathrm{ml}$ and irradiated using a Cs-137 $\gamma$ irradiation source with a Gammacell 3000. For i.v. immunisations, sporozoites were diluted to $40 \times 10^{4}$ sporozoites $/ \mathrm{ml}$ in Schneider's Drosophila medium and $100 \mu \mathrm{l}$ injected per mouse. For intramuscular immunisations prep was diluted to $40 \times 10^{4}$ sporozoites/ $\mathrm{ml}$ of Schneider's media and mixed with equal volumes of AddaVax adjuvant (InvivoGen). Balb/c mice were immunised intramuscular with $50 \mu \mathrm{l}$ per site sporozoite/adjuvant mixture at 0,3 , and $5 \mathrm{wk}$. The mice were challenged with five PbANKA 2.34 or PbANKA-PfCSP chimeric infected mosquito bites 1 wk after booster immunisation by allowing the mosquitoes to feed on the abdomen of each mouse for $15 \mathrm{~min}$. The salivary glands from all blood-fed mosquitoes have been dissected after the bites to confirm the presence of infective sporozoites. Since day 4 post challenge, the immunised mice were checked daily for the presence of $P$. berghei blood stages by microscopic examination of Giemsa-stained thin smears of tail 
blood and time to $1 \%$ parasitaemia determined by linear regression as above. The mouse was classified as negative for infection when no blood stages of parasite were present on day 14 after challenge.

\section{ELISAs}

Sporozoite lysate was prepared by pelleting MAF purified sporozoites and flash freezing, before diluting in PBS and using to coat 96-well immunosorbent plates (NUNC MaxiSorp) overnight (1,500 sporozoites per well). Subsequently, liquid was removed and wells allowed to air dry before blocking with $1 \%$ BSA in PBS. Wells were subsequently incubated with mouse serum with starting dilutions of 1:50 or 1:100 in $0.01 \%$ Tween-PBS. Anti-mouse IgG secondary antibody conjugated to AP (Sigma-Aldrich) was added after five washes in $0.01 \%$ Tween-PBS. AP was quantified after five washes in 0.01\% Tween-PBS using 4-nitrophenyl phosphate disodium salt hexahydrate (Sigma-Aldrich) measured at $405 \mathrm{~nm}$ absorbance.

\section{In vitro hepatocyte infection}

In vitro P. berghei sporozoite infections were carried out on HepG2 or primary hepatocytes $24 \mathrm{~h}$ after plating. Cells were plated into either 48 or 24 well plates (MatTek Corporation) at seeding densities of 50,000 and 150,000 for HepG2 cells, respectively, and 75,000 and 200,000 for primary rat hepatocytes, respectively. 24-well plates (Glass bottom; MatTek Corporation) were used for microscopy and 48 wells for quantitative PCR. Sporozoites in $4^{\circ} \mathrm{C}$ complete Schneider's Drosophila media were diluted in pre-warmed $\left(37^{\circ} \mathrm{C}\right)$ complete DMEM (for HepG2; Sigma-Aldrich) or primary hepatocyte medium (for primary hepatocytes; Promocell) to achieve a desired ratio of sporozoite to hepatocyte (1:1 for qPCR; 50,000-75,000 sporozoite per well or 1:2 for microscopy; $75,000-100,000$ sporozoite per well) and the culture media was exchanged with the sporozoite media. Cell cultures were carefully returned to the incubator to prevent swirling and an uneven distribution of sporozoites. Media was then no-longer exchanged for the remainder of the experiment.

For $P$. falciparum infections, HC-04 cells (media composition according to Yang et al [73]) were plated on 96 well plates (MatTek Corporation) coated with a $1 \mu \mathrm{g} / \mathrm{cm}^{2}$ mix of collagen, fibronectin, and laminin (see above) at a seeding density of 17,000 cells per well. Sporozoites were added to cells at a ratio of 5:1 (i.e., 85,000 sporozoites per well). The plates were immediately spun down for $5 \mathrm{~min}$ at $1,650 \mathrm{~g}$, before being returned to the incubator. After $4 \mathrm{~h}$, the cells were washed once with PBS and fixed with $4 \%$ PFA.

\section{Ex vivo hepatocyte challenge}

Rats were i.v. challenged with 30 million GFP transgenic sporozoites and $14 \mathrm{~h}$ later hepatocytes extracted by liver perfusion (above). Infected (GFP positive) hepatocytes were sorted (MoFlo) and plated for up to $30 \mathrm{~h}$.

\section{Bacterial contaminant quantification}

To assess the sterility of each purification step, tryptic soya broth (Oxoid) was inoculated with samples normalised by MEQ and absorbance at $600 \mathrm{~nm}$ measured after $16 \mathrm{~h}$ incubation at $37^{\circ} \mathrm{C}$.
Alternatively, samples normalised by MEQ were serially diluted in PBS and spread on blood-agar plates incubated overnight at $37^{\circ} \mathrm{C}$. Negative growth was confirmed by a further 24-h incubation.

\section{Protein purity quantification}

For Western blotting, sample was lysed using radioimmunoprecipitation assay buffer with protease inhibitor cocktail (Sigma-Aldrich), protein concentration normalised using Pierce BCA protein assay kit (Thermo Fisher Scientific), and sample loaded onto a 12\% TGX SDS-PAGE gel using reducing Laemmli buffer and transferred by semi-dry transfer onto a polyvinylidene difluoride (PVDF) membrane (Bio-Rad Laboratories). P. berghei CSP protein was probed using the 3D11 monoclonal (48) and detected using HRP chemiluminescence. Total protein concentration of purified mosquito sample was assessed in SDS-PAGE gels using Pierce silver stain kit (Thermo Fisher Scientific) or in solution using a Pierce BCA protein assay kit (Thermo Fisher Scientific). Dot blots were conducted on FFE fractions by loading $200 \mu \mathrm{l}$ of each fraction onto a multiscreen-IP plate $(0.45 \mu \mathrm{M}$; Millipore) pre-activated with methanol and incubated overnight $\left(4^{\circ} \mathrm{C}\right)$ before probing and detection of anti-mosquito actin (A2066; Sigma-Aldrich) similar to Western blotting using HRP and ECL. Alternatively, protein contaminants were assessed using liquid chromatography tandem-mass spectrometry (LC-MS/MS) with prior sample preparation in $6 \mathrm{M}$ urea, $100 \mathrm{mM}$ tris ( $\mathrm{pH}$ 7.8), $5 \mathrm{mM}$ dithiothreitol, and $20 \mathrm{mM}$ iodoacetamide with subsequent trypsin digestion overnight and desalting. Mass spectrometry output data were analysed using the Mascot algorithm (V2.4) and UniProt database. All media used for protein assessment were protein free. Equivalent volumes were injected onto FFE and collected for each treatment and total protein in each fraction quantified.

\section{Flow cytometry}

Flow cytometry was carried out using an LSRII (Becton Dickson). Hepatocytes were washed three times in 1× PBS and removed by gentle cell scraping. Hepatocytes were gated for single cell using FSC-H versus FSC-A and mCherry-P. berghei-infected cells detected in the PE-Texas Red channel by comparing to APC channel auto fluorescence. Uninfected hepatocytes were run as controls. GFPexpressing $P$. berghei-infected primary hepatocytes were sorted using a MoFlo cytometer (Beckman) gated for GFP-positive single cells.

\section{Immunofluorescent staining}

Cells were fixed with $4 \%$ paraformaldehyde and permeabilised using 1\% Triton X-100. Before antibody incubation cells were blocked with $1 \%$ BSA and then probed with primary and then secondary antibodies in 1\% BSA for 1-2 h. Nuclear staining was carried using DAPI. For CSP in/ out staining fixed cells were probed with CSP antibody before and after permeabilisation.

\section{Fluorescent microscopy}

Imaging of mCherry fluorescent $P$. berghei infected primary hepatocytes was carried out using $1.5 \mathrm{~mm}$ glass bottom dishes/ plates (Mattek) on a wide-field fluorescent microscope with LED 
fluorescence light source at $2 \mathrm{~Hz}$ using the Metamorph software package (Ludwig Institute). Infection numbers were determined by manual counting using the mCherry channel. Late-stage schizonts were captured using structured illumination microscopy with a Zeiss, Elyra (Imperial College London, FILM facility). P. falciparum-infected cells were imaged on a Nikon Eclipse Ti (Imperial College London, FILM facility). Image processing and analysis was automated by running a custom macro in Fiji. Quantification of intracellular versus extracellular parasites was carried by determining the area fraction (as a \%) of CSP in and outside of hepatocyte. Parasites were classed as inside cells if the area fraction of CSP outside and inside was $<10 \%$ and $>80 \%$, respectively. HC-04 numbers were quantified using nuclear count. Cell infection was calculated as the \% ratio of intracellular parasites to $\mathrm{HC}-04$ nuclei.

\section{Quantitative PCR}

DNA was extracted from cultures using phenol-chloroformisopropanol precipitation and re-suspended in molecular grade water. Nucleic acid concentration was determined using a Qubit fluorometer (Thermo Fisher Scientific). Quantification of P. berghei hepatocyte infection density based on absolute genome copies was determined using a standard curve plasmid containing a 271-bp fragment from murine heat shock protein (HSP) 60 (Ensembl: ENSMUST00000027123) housekeeping gene and a 176-bp fragment from P. berghei HSP70 gene (PBANKA_071190). 100 ng of DNA template was amplified using SsoAdvanced Universal SYBR green supermix (Bio-Rad Laboratories) run on a CFX Connect RT-PCR machine (Bio-Rad Laboratories) as per manufacturers standard protocol and parasite genome numbers determined using linear fit normalised to HSP60 housekeeping (HSP60 HepG2 F: GACCAAAGACGATGCCATGC, R: GCACAGCCACTCCATCTGAA; HSP60 Rat F: TGGAGAGGTCATCGTCACCA, R: CACAGCTACTCCATCTGAGAGT; HSP70 P. berghei F: AGGAATGCCAGGAGGAATGC, R: AGTTGGTCCACTTCCAGCTG).

\section{Animal research}

All animal works in this study were carried out according to the Animals (Scientific Procedures) Act 1986 Amendment Regulations 2012 (SI 2012/3039) with approval from the University of Oxford and Imperial College London Ethical Review Committee (PPL 30/2889 Oxford, 70/8788 and PDA3EBA4A Imperial). The Office of Laboratory Animal Welfare Assurance for Imperial College covers all Public Health Service supported activities involving live vertebrates in the US (no. A5634-01). Rats and mice were kept in individually ventilated cages.

\section{Statistical analysis}

Data were assessed for normality and equality of variance and used to determine the suitable statistical test as per John Tukey's exploratory data analysis method (74). Parametric data were assessed using a $t$ test and non-parametrically using a Mann-Whitney $U$ test for single treatment comparisons. Multiple treatments were compared using a t test with Bonferroni correction. Kaplan-Meier curves were compared using the Mantel-Cox test.

\section{Data Availability}

All data generated or analysed during this study are included in the manuscript and supporting files.

\section{Supplementary Information}

Supplementary Information is available at https://doi.org/10.26508/lsa. 202101094

\section{Acknowledgements}

This work is dedicated to the memory of Shahid M Kahn for his contributions to whole sporozoite vaccinology and for his generosity of spirit, collaborating on this work. We thank Alex Fyfe and Mark Tunnicliff for maintaining the mosquito colony, Andrew Worth for help with FACS sorting, Mark Shipman and Andreas Bruckbauer for help with microscopy, and Stephen Rothery for writing the script for automated image analysis. We also thank Annie Yang for advice on $P$. falciparum in vitro hepatocyte assays. We gratefully acknowledge Infravec for supporting our $P$. falciparum research, in particular Roel Heutink and Geert-Jan Van for provision of infected mosquitoes required in the testing phases of this work. Research was directly supported by Wellcome (Investigator Award 100993/Z/13/Z, J Baum and Career Development Fellowship 097395/Z/11/Z, A Reyes-Sandoval), the Bill \& Melinda Gates Foundation (OPP1200274, J Baum), and an Medical Research Concil (MRC) research training and support grant (1240480, J Blight). AM Blagborough thanks the MRC (New Investigator Research grant MR/N00227X/1), PATH-MVI, Isaac Newton Trust, Wellcome Trust Institutional Strategic Support Fund and, University of Cambridge Junior Research Fellowship Scheme for funding. E Alves was funded by CAPES (Program Science without Borders, CSF-2361/13-2). FM Ashcroft and H Kramer thank Wellcome (OXION Strategic award 084655/Z/08/Z) for support. Microscopy work was supported through Mark Shipman in the Ludwig Institute at Oxford and the Facility for Imaging by Light Microscopy at Imperial College London, supported by previous funding from Wellcome (grant 104931/Z/14/Z), and the Biotechnology and Biological Sciences Research Council, UK (grant BB/L015129/1). This project has received resources funded by the European Union's Horizon 2020 research and innovation program under grant agreement No 731060 (Infravec2)

\section{Author Contributions}

J Blight: conceptualization, data curation, formal analysis, supervision, validation, investigation, visualization, methodology, project administration, and writing-original draft, review, and editing. KA Sala: data curation, validation, investigation, and writingoriginal draft, review, and editing.

E Atcheson: data curation, investigation, and writing-review and editing.

H Kramer: data curation, investigation, and writing-review and editing.

A El-Turabi: data curation, investigation, and writing-review and editing.

E Real: data curation, investigation, methodology, and writing-review and editing.

FA Dahalan: data curation, investigation, and writing-review and editing.

P Bettencourt: data curation, supervision, and writing-review and editing. 
E Dickinson-Craig: investigation and writing-review and editing.

E Alves: investigation and writing-review and editing.

AM Salman: investigation and writing-review and editing.

C) Janse: funding acquisition, investigation, project administration, and writing-review and editing.

F Ashcroft: resources, supervision, funding acquisition, project administration, and writing-review and editing.

AVS Hill: supervision, funding acquisition, project administration, and writing-review and editing.

A Reyes-Sandoval: resources, supervision, funding acquisition, project administration, and writing-review and editing.

AM Blagborough: resources, supervision, funding acquisition, investigation, methodology, project administration, and writingoriginal draft, review, and editing.

J Baum: conceptualization, resources, data curation, supervision, funding acquisition, visualization, project administration, and writing-original draft, review, and editing.

\section{Conflict of Interest Statement}

The authors declare that they have no conflict of interest.

\section{References}

1. WHO (2019) World Malaria Report. Geneva: Switzerland: WHO.

2. Duffy PE, Patrick Gorres J (2020) Malaria vaccines since 2000: Progress, priorities, products. NPJ Vaccin 5: 48. doi:10.1038/s41541-020-0196-3

3. Chatterjee D, Cockburn IA (2021) The challenges of a circumsporozoite protein-based malaria vaccine. Expert Rev Vaccin 20: 113-125. doi:10.1080/14760584.2021.1874924

4. RTS,S Clinical Trials Partnership (2015) Efficacy and safety of RTS,S/AS01 malaria vaccine with or without a booster dose in infants and children in Africa: Final results of a phase 3 , individually randomised, controlled trial. Lancet 386: 31-45. doi:10.1016/S0140-6736(15)60721-8

5. Roestenberg M, McCall M, Hopman J, Wiersma J, Luty AJ, van Gemert GJ, van de Vegte-Bolmer M, van Schaijk B, Teelen K, Arens T, et al (2009) Protection against a malaria challenge by sporozoite inoculation. $N$ Engl J Med 361: 468-477. doi:10.1056/NEJMoa0805832

6. Clyde DF, Most H, McCarthy VC, Vanderberg JP (1973) Immunization of man against sporozite-induced falciparum malaria. Am J Med Sci 266 169-177. doi:10.1097/00000441-197309000-00002

7. Rieckmann KH, Carson PE, Beaudoin RL, Cassells JS, Sell KW (1974) Letter: Sporozoite induced immunity in man against an Ethiopian strain of Plasmodium falciparum. Trans R Soc Trop Med Hyg 68: 258-259. doi:10.1016/0035-9203(74)90129-1

8. Hoffman SL, Goh LM, Luke TC, Schneider I, Le TP, Doolan DL, Sacci J, de la Vega P, Dowler M, Paul C, et al (2002) Protection of humans against malaria by immunization with radiation-attenuated Plasmodium falciparum sporozoites. J Infect Dis 185: 1155-1164. doi:10.1086/339409

9. Epstein JE, Paolino KM, Richie TL, Sedegah M, Singer A, Ruben AJ, Padilla D, Saverino E, Murshedkar T, Gunasekera A, et al (2017) Protection against Plasmodium falciparum malaria by PfSPZ vaccine. JCI Insight 2: e89154. doi:10.1172/jci.insight.89154

10. Goswami D, Betz W, Locham NK, Parthiban C, Brager C, Schafer C, Camargo N, Nguyen T, Kennedy SY, Murphy SC, et al (2020) A replicationcompetent late liver stage-attenuated human malaria parasite. $\mathrm{JCl}$ Insight 5: e135589. doi:10.1172/jci.insight.135589
11. Sigler $\mathrm{Cl}$, Leland $\mathrm{P}$, Hollingdale MR (1984) In vitro infectivity of irradiated Plasmodium berghei sporozoites to cultured hepatoma cells. Am I Trop Med Hyg 33: 544-547. doi:10.4269/ajtmh.1984.33.544

12. Nüssler A, Follezou JY, Miltgen F, Mazier D (1989) Effect of irradiation on Plasmodium sporozoites depends on the species of hepatocyte infected. Trop Med Parasitol 40: 468-469.

13. Hoffman SL, Billingsley PF, James E, Richman A, Loyevsky M, Li T, Chakravarty S, Gunasekera A, Chattopadhyay R, Li M, et al (2010) Development of a metabolically active, non-replicating sporozoite vaccine to prevent Plasmodium falciparum malaria. Hum Vaccin 6 : 97-106. doi:10.4161/hv.6.1.10396

14. Sissoko MS, Healy SA, Katile A, Omaswa F, Zaidi I, Gabriel EE, Kamate B, Samake Y, Guindo MA, Dolo A, et al (2017) Safety and efficacy of PfSPZ vaccine against Plasmodium falciparum via direct venous inoculation in healthy malaria-exposed adults in Mali: A randomised, double-blind phase 1 trial. Lancet Infect Dis 17: 498-509. doi:10.1016/S1473-3099(17) 30104-4

15. Jongo SA, Shekalaghe SA, Church LWP, Ruben AJ, Schindler T, Zenklusen I, Rutishauser T, Rothen J, Tumbo A, Mkindi C, et al (2018) Safety, immunogenicity, and protective efficacy against controlled human malaria infection of Plasmodium falciparum sporozoite vaccine in Tanzanian adults. Am J Trop Med Hyg 99: 338-349. doi:10.4269/ajtmh.171014

16. WHO (2015) Preferred Product Characteristics for Malaria Vaccines. Geneva, Switzerland: World Health Organisation.

17. March S, Ng S, Velmurugan S, Galstian A, Shan J, Logan DJ, Carpenter AE, Thomas D, Sim BK, Mota MM, et al (2013) A microscale human liver platform that supports the hepatic stages of Plasmodium falciparum and vivax. Cell Host Microbe 14: 104-115. doi:10.1016/j.chom.2013.06.005

18. Mazier D, Beaudoin RL, Mellouk S, Druilhe P, Texier B, Trosper J, Miltgen F, Landau I, Paul C, Brandicourt O, et al (1985) Complete development of hepatic stages of Plasmodium falciparum in vitro. Science 227: 440-442. doi:10.1126/science.3880923

19. Corradetti A, Verolini F, Sebastiani A, Proietti AM, Amati L (1964) Fluorescent antibody testing with sporozoites of plasmodia. Bull World Health Organ 30: 747-750.

20. Sattabongkot J, Yimamnuaychoke N, Leelaudomlipi S, Rasameesoraj M Jenwithisuk R, Coleman RE, Udomsangpetch R, Cui L, Brewer TG (2006) Establishment of a human hepatocyte line that supports in vitro development of the exo-erythrocytic stages of the malaria parasites Plasmodium falciparum and P. vivax. Am J Trop Med Hyg 74: 708-715. doi:10.4269/ajtmh.2006.74.708

21. Hollingdale MR, Collins WE, Campbell CC, Schwartz AL (1985) In vitro culture of two populations (dividing and nondividing) of exoerythrocytic parasites of Plasmodium vivax. Am J Trop Med Hyg 34: 216-222. doi:10.4269/ajtmh.1985.34.216

22. Zou X, House BL, Zyzak MD, Richie TL, Gerbasi VR (2013) Towards an optimized inhibition of liver stage development assay (ILSDA) for Plasmodium falciparum. Malar J 12: 394. doi:10.1186/1475-2875-12-394

23. Lupton EJ, Roth A, Patrapuvich R, Maher SP, Singh N, Sattabongkot J, Adams JH (2015) Enhancing longevity of Plasmodium vivax and P. falciparum sporozoites after dissection from mosquito salivary glands. Parasitol Int 64: 211-218. doi:10.1016/j.parint.2014.11.016

24. Kennedy M, Fishbaugher ME, Vaughan AM, Patrapuvich R, Boonhok R, Yimamnuaychok N, Rezakhani N, Metzger P, Ponpuak M, Sattabongkot J, et al (2012) A rapid and scalable density gradient purification method for Plasmodium sporozoites. Malar J 11: 421. doi:10.1186/1475-2875-11-421

25. Schleicher TR, Yang J, Freudzon M, Rembisz A, Craft S, Hamilton M, Graham M, Mlambo G, Tripathi AK, Li Y, et al (2018) A mosquito salivary gland protein partially inhibits Plasmodium sporozoite cell traversal and transmission. Nat Commun 9: 2908. doi:10.1038/s41467-018-05374-3

26. Vogt MB, Lahon A, Arya RP, Kneubehl AR, Spencer Clinton JL, Paust S, Rico-Hesse R (2018) Mosquito saliva alone has profound effects on the 
human immune system. PLoS Negl Trop Dis 12: e0006439. doi:10.1371/ journal.pntd.0006439

27. Coppi A, Natarajan R, Pradel G, Bennett BL, James ER, Roggero MA, Corradin G, Persson C, Tewari R, Sinnis P (2011) The malaria circumsporozoite protein has two functional domains, each with distinct roles as sporozoites journey from mosquito to mammalian host. J Exp Med 208: 341-356. doi:10.1084/jem.20101488

28. Ishino T, Chinzei Y, Yuda M (2005) Two proteins with 6-cys motifs are required for malarial parasites to commit to infection of the hepatocyte. Mol Microbiol 58: 1264-1275. doi:10.1111/j.1365-2958.2005.04801.x

29. Ishino T, Yano K, Chinzei Y, Yuda M (2004) Cell-passage activity is required for the malarial parasite to cross the liver sinusoidal cell layer. PLOS Biol 2: E4. doi:10.1371/journal.pbio.0020004

30. Sato Y, Montagna GN, Matuschewski K (2014) Plasmodium berghei sporozoites acquire virulence and immunogenicity during mosquito hemocoel transit. Infect Immun 82: 1164-1172. doi:10.1128/IAI.00758-13

31. Kariu T, Ishino T, Yano K, Chinzei Y, Yuda M (2006) CelTOS, a novel malarial protein that mediates transmission to mosquito and vertebrate hosts. Mol Microbiol 59: 1369-1379. doi:10.1111/j.13652958.2005.05024.X

32. Hollingdale MR, Nardin EH, Tharavanij S, Schwartz AL, Nussenzweig RS (1984) Inhibition of entry of Plasmodium falciparum and P. vivax sporozoites into cultured cells; an in vitro assay of protective antibodies. J Immunol 132: 909-913.

33. Siau A, Silvie O, Franetich JF, Yalaoui S, Marinach C, Hannoun L, van Gemert G-J, Luty AJF, Bischoff E, David PH, et al (2008) Temperature shift and host cell contact up-regulate sporozoite expression of Plasmodium falciparum genes involved in hepatocyte infection. PLoS Pathog 4: e1000121. doi:10.1371/journal.ppat.1000121

34. Ozaki LS, Gwadz RW, Godson GN (1984) Simple centrifugation method for rapid separation of sporozoites from mosquitoes. J Parasitol 70: 831-833. doi:10.2307/3281779

35. Bosworth AB, Schneider I, Freier JE (1975) Mass isolation of Anopheles stephensi salivary glands infected with malarial sporozoites. J Parasitol 61: 769-772. doi:10.2307/3279485

36. Krettli A, Chen DH, Nussenzweig RS (1973) Immunogenicity and infectivity of sporozoites of mammalian malaria isolated by density-gradient centrifugation. J Protozool 20: 662-665. doi:10.1111/j.15507408.1973.tb03594.x

37. Beaudoin RL, Strome CP, Mitchell F, Tubergen TA (1977) Plasmodium berghei: Immunization of mice against the ANKA strain using the unaltered sporozoite as an antigen. Exp Parasitol 42: 1-5. doi:10.1016/ 0014-4894(77)90054-6

38. Pacheco ND, Strome CP, Mitchell F, Bawden MP, Beaudoin RL (1979) Rapid, large-scale isolation of Plasmodium berghei sporozoites from infected mosquitoes. J Parasitol 65: 414-417. doi:10.2307/3280286

39. Wood DE, Smrkovski LL, McConnell E, Pacheco ND, Bawden MP (1979) The use of membrane screen filters in the isolation of Plasmodium berghei sporozoites from mosquitos. Bull World Health Organ 57: 69-74.

40. Schulman S, Oppenheim JD, Vanderberg JP (1980) Plasmodium berghei and Plasmodium knowlesi: Serum binding to sporozoites. Exp Parasitol 49: 420-429. doi:10.1016/0014-4894(80)90076-4

41. Moser G, Brohn FH, Danforth HD, Nussenzweig RS (1978) Sporozoites of rodent and simian malaria, purified by anion exchangers, retain their immunogenicity and infectivity. J Protozool 25: 119-124. doi:10.1111/ j.1550-7408.1978.tb03881.x

42. Mack SR, Vanderberg JP, Nawrot R (1978) Column separation of Plasmodium berghei sporozoites. J Parasitol 64: 166-168. doi:10.2307/ 3279638

43. Heidrich HG, Danforth HD, Leef JL, Beaudoin RL (1983) Free-flow electrophoretic separation of Plasmodium berghei sporozoites. J Parasitol 69: 360-367. doi:10.2307/3281235
44. Hannig K, Heidrich HG (1989), 1st ed. edn Free Flow Electrophoresis: An Important Analytical and Preparative Method of Biology, Biochemistry and Diagnostics. Darmstadt, Germany: GIT Verlag, Ernst Giebeler.

45. Schrum M, Canezin A, Chakravarty S, Laskowski M, Comert S, Sevimli Y, Chirikjian GS, Hoffman SL, Taylor RH (2019) An efficient production process for extracting salivary glands from mosquitoes arXiv.190302532.

46. Blagborough AM, Churcher TS, Upton LM, Ghani AC, Gething PW, Sinden RE (2013) Transmission-blocking interventions eliminate malaria from laboratory populations. Nat Commun 4: 1812. doi:10.1038/ ncomms 2840

47. Kramer LD, Vanderberg JP (1975) Intramuscular immunization of mice with irradiated Plasmodium berghei sporozoites. Enhancement of protection with albumin. Am J Trop Med Hyg 24: 913-916. doi:10.4269/ ajtmh.1975.24.913

48. Yoshida N, Nussenzweig RS, Potocnjak P, Nussenzweig V, Aikawa M (1980) Hybridoma produces protective antibodies directed against the sporozoite stage of malaria parasite. Science 207: 71-73. doi:10.1126/ science.6985745

49. Ferguson DJ, Balaban AE, Patzewitz EM, Wall RJ, Hopp CS, Poulin B, Mohmmed A, Malhotra P, Coppi A, Sinnis P, et al (2014) The repeat region of the circumsporozoite protein is critical for sporozoite formation and maturation in Plasmodium. PLoS One 9: e113923. doi:10.1371/ journal.pone. 0113923

50. Winn WC, Koneman EW (2016) Koneman's Color Atlas and Textbook of Diagnostic Microbiology. Philadelphia, PA: Lippincott Williams and Wilkins.

51. Galarza DA, Lopez-Sebastian A, Woelders H, Blesbois E, SantiagoMoreno J (2018) Sephadex filtration as successful alternative to densitygradient centrifugation procedures for ram sperm selection with improved kinetics. Anim Reprod Sci 192: 261-270. doi:10.1016 j.anireprosci.2018.03.022

52. Hegge S, Kudryashev M, Smith A, Frischknecht F (2009) Automated classification of Plasmodium sporozoite movement patterns reveals a shift towards productive motility during salivary gland infection. Biotechnol J 4: 903-913. doi:10.1002/biot.200900007

53. Collins KA, Snaith R, Cottingham MG, Gilbert SC, Hill AVS (2017) Enhancing protective immunity to malaria with a highly immunogenic virus-like particle vaccine. Sci Rep 7: 46621. doi:10.1038/srep46621

54. Kaiser K, Camargo N, Kappe SH (2003) Transformation of sporozoites into early exoerythrocytic malaria parasites does not require host cells. J Exp Med 197: 1045-1050. doi:10.1084/jem.20022100

55. Gego A, Silvie O, Franetich JF, Farhati K, Hannoun L, Luty AJ, Sauerwein RW, Boucheix C, Rubinstein E, Mazier D (2006) New approach for highthroughput screening of drug activity on Plasmodium liver stages. Antimicrob Agents Chemother 50: 1586-1589. doi:10.1128/AAC.50.4.15861589

56. Yahiya S, Rueda-Zubiaurre A, Delves MJ, Fuchter MJ, Baum J (2019) The antimalarial screening landscape-looking beyond the asexual blood stage. Curr Opin Chem Biol 50: 1-9. doi:10.1016/j.cbpa.2019.01.029

57. Vanderberg JP (1975) Development of infectivity by the Plasmodium berghei sporozoite. J Parasitol 61: 43-50. doi:10.2307/3279102

58. Billman ZP, Seilie AM, Murphy SC (2016) Purification of Plasmodium sporozoites enhances parasite-specific CD8+ T cell responses. Infect Immun 84: 2233-2242. doi:10.1128/IAI.01439-15

59. Patel H, Althubaiti N, Parmar R, Yadav N, Joshi U, Tyagi RK, Krzych U, Dalai SK (2019) Parasite load stemming from immunization route determines the duration of liver-stage immunity. Parasite Immunol 41: e12622. doi:10.1111/pim.12622

60. Lasonder E, Janse CJ, van Gemert GJ, Mair GR, Vermunt AM, Douradinha BG, van Noort V, Huynen MA, Luty AJF, Kroeze H, et al (2008) Proteomic profiling of Plasmodium sporozoite maturation identifies new proteins essential for parasite development and infectivity. PLOS Pathog 4: e1000195. doi:10.1371/journal.ppat.1000195 
61. Lindner SE, Swearingen KE, Shears MJ, Walker MP, Vrana EN, Hart KJ, Minns AM, Sinnis P, Moritz RL, Kappe SHI (2019) Transcriptomics and proteomics reveal two waves of translational repression during the maturation of malaria parasite sporozoites. Nat Commun 10: 4964. doi:10.1038/s41467-019-12936-6

62. Roestenberg M, Walk J, van der Boor SC, Langenberg MCC, Hoogerwerf MA, Janse JJ, Manurung M, Yap XZ, García AF, Koopman JPR, et al (2020) A double-blind, placebo-controlled phase 1/2a trial of the genetically attenuated malaria vaccine PfSPZ-GA1. Sci Transl Med 12: eaaz5629. doi:10.1126/scitranslmed.aaz5629

63. Reuling IJ, Mendes AM, de Jong GM, Fabra-Garcia A, Nunes-Cabaco H, van Gemert GJ, Graumans W, Coffeng LE, de Vlas SJ, Yang ASP, et al (2020) An open-label phase $1 / 2$ a trial of a genetically modified rodent malaria parasite for immunization against Plasmodium falciparum malaria. Sci Transl Med 12: eaay2578. doi:10.1126/scitranslmed.aay2578

64. Lin JW, Annoura T, Sajid M, Chevalley-Maurel S, Ramesar J, Klop O, Franke-Fayard BMD, Janse CJ, Khan SM (2011) A novel "ene insertion/ marker out" (GIMO) method for transgene expression and gene complementation in rodent malaria parasites. PLoS One 6: e29289. doi:10.1371/journal.pone.0029289

65. Hopp CS, Chiou K, Ragheb DR, Salman AM, Khan SM, Liu AJ, Sinnis P (2015) Longitudinal analysis of Plasmodium sporozoite motility in the dermis reveals component of blood vessel recognition. elife 4: e07789. doi:10.7554/elife.07789

66. Mueller AK, Camargo N, Kaiser K, Andorfer C, Frevert U, Matuschewski K, Kappe SHI (2005) Plasmodium liver stage developmental arrest by depletion of a protein at the parasite-host interface. Proc Natl Acad Sci U S A 102: 3022-3027. doi:10.1073/pnas.0408442102

67. Salman AM, Mogollon CM, Lin JW, van Pul FJ, Janse CJ, Khan SM (2015) Generation of transgenic rodent malaria parasites expressing human malaria parasite proteins. Methods Mol Biol 1325: 257-286. doi:10.1007/ 978-1-4939-2815-6_21

68. Janse CJ, Ramesar J, Waters AP (2006) High-efficiency transfection and drug selection of genetically transformed blood stages of the rodent malaria parasite Plasmodium berghei. Nat Protoc 1: 346-356. doi:10.1038/nprot.2006.53

69. Sumitani M, Kasashima K, Yamamoto DS, Yagi K, Yuda M, Matsuoka H, Yoshida S (2013) Reduction of malaria transmission by transgenic mosquitoes expressing an antisporozoite antibody in their salivary glands. Insect Mol Biol 22: 41-51. doi:10.1111/j.1365-2583.2012.01168.x

70. Delves MJ, Straschil U, Ruecker A, Miguel-Blanco C, Marques S, Dufour AC, Baum J, Sinden RE (2016) Routine in vitro culture of P. falciparum gametocytes to evaluate novel transmission-blocking interventions. Nat Protoc 11: 1668-1680. doi:10.1038/nprot.2016.096

71. Ingber DE (1990) Fibronectin controls capillary endothelial cell growth by modulating cell shape. Proc Natl Acad Sci U S A 87: 3579-3583. doi:10.1073/pnas.87.9.3579

72. Kostadinova R, Boess $F$, Applegate $D$, Suter $L$, Weiser $T$, Singer $T$, Naughton B, Roth A (2013) A long-term three dimensional liver coculture system for improved prediction of clinically relevant druginduced hepatotoxicity. Toxicol Appl Pharmacol 268: 1-16. doi:10.1016/ j.taap.2013.01.012

73. Yang ASP, van Waardenburg YM, van de Vegte-Bolmer M, van Gemert GA, Graumans W, de Wilt JHW, Sauerwein RW (2021) Zonal human hepatocytes are differentially permissive to Plasmodium falciparum malaria parasites. EMBO J 40: e106583. doi:10.15252/embj.2020106583

74. Tukey JW (1977) Exploratory Data Analysis, $1^{\text {st }}$ ed. London, UK: Pearson.

75. Angrisano F, Riglar DT, Sturm A, Volz JC, Delves MJ, Zuccala ES, Turnbull L, Dekiwadia C, Olshina MA, Marapana DS, et al (2012) Spatial localisation of actin filaments across developmental stages of the malaria parasite. PLoS One 7: e32188. doi:10.1371/journal.pone.0032188

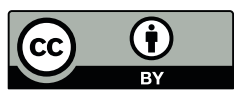

License: This article is available under a Creative Commons License (Attribution 4.0 International, as described at https://creativecommons.org/ licenses/by/4.0/). 